\title{
Long knots and maps between operads
}

\author{
WILLIAM DWYER \\ KATHRYN HESS
}

\begin{abstract}
We identify the space of tangentially straightened long knots in $\mathbb{R}^{m}, m \geq 4$, as the double loops on the space of derived operad maps from the associative operad into a version of the little $m$-disk operad. This verifies a conjecture of Kontsevich, Lambrechts and Turchin.
\end{abstract}

18D50, 55P48; 18G55, 57Q45

\section{Introduction}

A long knot in Euclidean $m$-space $\mathbb{R}^{m}$ is a smooth embedding $\mathbb{R} \rightarrow \mathbb{R}^{m}$ which agrees with inclusion of the first coordinate axis on the complement of some compact set in $\mathbb{R}$; a tangential straightening for the knot is a null homotopy (constant near $\infty$ ) of the map $\mathbb{R} \rightarrow S^{m-1}$ obtained by taking the unit tangent vector of the knot at each point. See Sinha $[23,5.1]$ for more details. Starting from work of Goodwillie, Klein, and Weiss $[9 ; 10 ; 11 ; 24]$ on embedding spaces, Sinha $[23]$ has proved that the space of tangentially straightened long knots in $\mathbb{R}^{m}$ is equivalent to the homotopy limit of an explicit cosimplicial space constructed from the $m$-th Kontsevich operad $\mathcal{K}_{m}$.

Let $\mathcal{A}$ denote the associative operad. The cosimplicial space Sinha considers is derived by formulas of McClure and Smith [19] from an operad map $\mathcal{A} \rightarrow \mathcal{K}_{m}$; more generally, McClure and Smith build a cosimplicial space $\mathcal{O}^{\bullet}$ from any operad $\mathcal{O}$ of spaces and operad map $\mathcal{A} \rightarrow \mathcal{O}$. Our main theorem gives an independent formula for the homotopy limit of this construction. Say that the operad $\mathcal{O}$ is reduced if $\mathcal{O}_{0}$ and $\mathcal{O}_{1}$ are weakly contractible.

1.1 Theorem (Section 8) Let $\mathcal{O}$ be a reduced operad of simplicial sets, $\omega: \mathcal{A} \rightarrow \mathcal{O}$ an operad map, and $\mathcal{O}^{\bullet}$ the cosimplicial object associated to $\omega$ by [19]. Then there is a natural weak homotopy equivalence

$$
\operatorname{holim} \mathcal{O}^{\bullet} \sim \Omega^{2} \operatorname{Map}^{\mathrm{h}}(\mathcal{A}, \mathcal{O})_{\omega} .
$$


The space on the right is the double loops, based at $\omega$, on a derived space of operad maps $\mathcal{A} \rightarrow \mathcal{O}$. See below for more details. Let $\operatorname{Sing}(-)$ be the singular complex functor. Since $\mathcal{K}_{m}$ is reduced, applying Theorem 1.1 with $\mathcal{O}=\operatorname{Sing}\left(\mathcal{K}_{m}\right)$ shows that the space of tangentially straightened long knots in $\mathbb{R}^{m}$ is weakly homotopy equivalent to the double loop space on a space of operad maps from the associative operad to $\operatorname{Sing}\left(\mathcal{K}_{m}\right)$. This verifies a conjecture of Kontsevich (see Sinha [23, $\left.2.17 \mathrm{ff}\right]$ ) as adjusted by Lambrechts and Turchin and re-expressed simplicially. The operad $\mathcal{K}_{m}$ is a pared-down model of the little $m$-disk operad; see Kontsevich [14] and Sinha [23, Section 4]. A result similar to Theorem 1.1 has been announced recently by Lambrechts and Turchin.

Theorem 1.1 is derived from a very general result about monoids and bimodules in a not-necessarily-symmetric monoidal category; see Theorem 1.7 or 3.11. This result is relevant to (and sometimes familiar in) many different contexts; see Section 1.13.

1.2 More details From this point on in the paper "space" means "simplicial set." Let $\mathbf{S p}$ denote the category of spaces and $\mathbf{S}$ the category of graded spaces (sequences of spaces indexed by the nonnegative integers). The composition product $X \circ Y$ of graded spaces is given by

$$
(X \circ Y)_{n}=\coprod_{i, j_{1}+\cdots j_{i}=n} X_{i} \times Y_{j_{1}} \times \cdots \times Y_{j_{i}} .
$$

An operad is a monoid object for this associative (but nonsymmetric) product. See May [17] or Sinha [23, 2.13], but note that we do not impose constraints on an operad in levels 0 and 1 . The associative operad $\mathcal{A}$ is the graded space which has a single point at every level $n, n \geq 0$; it has a unique operad structure. We will let $\mathbf{O}$ denote the category of operads.

The category $\mathbf{S}$ is a simplicial model category in which a map $X \rightarrow Y$ is an equivalence, cofibration, or fibration if and only if each individual map $X_{n} \rightarrow Y_{n}$ has the corresponding property in the usual model structure on spaces; see Hovey [13, 3.2]. It follows from Rezk's thesis [21] (see also Rezk [22, Proof of 7.2]) that $\mathbf{O}$ is a simplicial model category in which a map $\mathcal{P} \rightarrow \mathcal{Q}$ is an equivalence or a fibration if and only if the underlying map in $\mathbf{S}$ has the corresponding property. The derived mapping space $\operatorname{Map}^{\mathrm{h}}(\mathcal{A}, \mathcal{O})$ in Theorem 1.1 is the simplicial mapping space $\operatorname{Map}_{\mathbf{o}}\left(\mathcal{A}^{\mathrm{c}}, \mathcal{O}^{\mathrm{f}}\right)$, where $\mathcal{A}^{\mathrm{c}}$ is a cofibrant replacement for $\mathcal{A}$ and $\mathcal{O}^{\mathrm{f}}$ a fibrant replacement for $\mathcal{O}$.

In describing the left hand side of Theorem 1.1, it is convenient to write a point $f \in \mathcal{O}_{i}$ as if it were a function $f\left(x_{1}, \ldots, x_{i}\right)$ of $i$ formal variables. If the image $h$ of a tuple $\left(f, g_{1}, \ldots, g_{i}\right)$ under the operad structure map $\mathcal{O} \circ \mathcal{O} \rightarrow \mathcal{O}$ is written as a composite

$$
h\left(x_{1}, \ldots, x_{J}\right)=f\left(g_{1}\left(x_{1}, \ldots, x_{j_{1}}\right), \ldots, g_{i}\left(x_{J-j_{i}+1}, \ldots, x_{J}\right)\right)
$$


(where $g_{k} \in \mathcal{O}_{j_{k}}$ and $J=\sum j_{k}$ ), then the operad identities for the structure map express the associativity of composition. (Writing $h$ as a composite in this way amounts to pretending for the sake of notation that $\mathcal{O}$ is the endomorphism operad of a space.) Given a map $\mathcal{A} \rightarrow \mathcal{O}$, let $\epsilon \in \mathcal{O}_{0}$ and $\mu \in \mathcal{O}_{2}$ be the images respectively of $\mathcal{A}_{0} \rightarrow \mathcal{O}_{0}$ and $\mathcal{A}_{2} \rightarrow \mathcal{O}_{2}$. We will think of $\mu$ as a multiplication and temporarily write $x * y$ for $\mu(x, y)$. For each $n \geq 0$ the cosimplicial space $\mathcal{O}^{\bullet}$ has $\mathcal{O}_{n}$ in cosimplicial degree $n$; the coface and codegeneracy operators on $f \in \mathcal{O}_{n}$ are given by

$$
\begin{aligned}
\left(d^{i} f\right)\left(x_{1}, \ldots, x_{n+1}\right) & = \begin{cases}x_{1} * f\left(x_{2}, \ldots, x_{n+1}\right) & i=0, \\
f\left(x_{i}, \ldots, x_{i} * x_{i+1}, \ldots, x_{n+1}\right) & 1 \leq i \leq n, \\
f\left(x_{1}, \ldots, x_{n}\right) * x_{n+1} & i=n+1,\end{cases} \\
\left(s_{i} f\right)\left(x_{1}, \ldots x_{n-1}\right) & =f\left(x_{1}, \ldots, x_{i-1}, \epsilon, x_{i}, \ldots, x_{n-1}\right) .
\end{aligned}
$$

For other descriptions of $\mathcal{O}^{\bullet}$ see [23, 2.17] or [19, Section 3].

1.4 Method of proof Oddly enough, our proof of Theorem 1.1 comes down to applying a single principle twice, in very different cases; each application gives rise to one instance of $\Omega$. Before looking at these applications, we will describe the principle.

1.5 A connection between maps and bimodules Suppose that $\left(\mathbf{C}, \diamond, e_{\diamond}\right)$ is a category with a monoidal product $\diamond$ for which $e_{\diamond}$ is the unit, and that $\mathbf{M}$ is the category of monoids in $\mathbf{C}$. It is not necessary for $\diamond$ to be symmetric monoidal. Given monoids $R, S$ in $\mathbf{C}$, the notions of left $R$-module, right $S$-module, and $R-S$-bimodule are defined as usual. A pointed module or bimodule $X$ is one which is provided with a C-map $e_{\diamond} \rightarrow X$; a monoid under $R-S$ is a monoid $T$ together with monoid maps $R \rightarrow T$ and $S \rightarrow T$.

Suppose that there is a model category structure on $\mathbf{C}$ which induces compatible (Section 3) model structures on all monoid and bimodule categories. There is a forgetful functor $\llbracket$ from monoids under $R-S$ to pointed $R-S$-bimodules, with the basepoint for $\llbracket T$ given by the unit map $e_{\diamond} \rightarrow T$. Assume that $\downarrow$ has a left adjoint $E$ (the enveloping monoid functor) with the property that $(E, \downarrow)$ forms a Quillen pair, and let $E^{\mathrm{h}}$ denote the left derived functor (Remark 3.3) of $E$.

Say that a pointed right $S$-module $X$ is distinguished if the map

$$
S \cong e_{\diamond} \diamond S \rightarrow X \diamond S \rightarrow X
$$

induced by $e_{\diamond} \rightarrow X$ and $X \diamond S \rightarrow S$ is an equivalence; an $R-S$-bimodule is distinguished if it is distinguished as a right $S$-module. Similarly a monoid $T$ under $R-S$ is distinguished if $T$ is distinguished as a right $S$-module, or equivalently, if the structure map $S \rightarrow T$ is an equivalence. There is one major axiom: 
1.6 Axiom $\mathbf{E}$ If $X$ is a distinguished $R-S$-bimodule, then $E^{\mathrm{h}}(X)$ is a distinguished monoid under $R-S$.

The next theorem is the source of $\Omega$.

1.7 Theorem (Theorem 3.11) Let $\omega: R \rightarrow S$ be a map of monoids in $\mathbf{C}$. Then under the above assumptions, and some additional technical conditions, there is a fibration sequence

$$
\Omega \operatorname{Map}_{\mathbf{M}}^{\mathrm{h}}(R, S)_{\omega} \rightarrow \operatorname{Map}_{R-R}^{\mathrm{h}}(R, S) \rightarrow \operatorname{Map}_{\mathbf{C}}^{\mathrm{h}}\left(e_{\diamond}, S\right)
$$

In this statement $\mathrm{Map}^{\mathrm{h}}$ stands for the general model category theoretic derived mapping space (Section 2.1), which agrees with the appropriate derived simplicial mapping space if the model category involved is a simplicial model category. The middle space is computed in the category of $R-R$-bimodules, with $S$ treated as an $R-R$-bimodule via $\omega$. The right hand map is induced by the unit map $e_{\diamond} \rightarrow R$, and the homotopy fibre is meant to be computed over the unit map $e_{\diamond} \rightarrow S$.

1.8 The first application of Theorem 1.7 Here $\left(\mathbf{C}, \diamond, e_{\diamond}\right)$ is $\left(\mathbf{S}, \circ, e_{\circ}\right)$; the unit $e_{\circ}$ is a graded space which is empty except for a single point at level 1 . The monoids in $\mathbf{C}$ are the operads. For any graded space $X$, the mapping space $\operatorname{Map}_{\mathbf{S}}^{\mathrm{h}}\left(e_{\circ}, X\right)$ is equivalent to $X_{1}$, and so Theorem 1.7 gives the following.

1.9 Theorem (Section 6) Suppose that $\omega: \mathcal{A} \rightarrow \mathcal{O}$ is a map of operads, with $\mathcal{O}_{1}$ contractible. Then there is an equivalence

$$
\Omega \operatorname{Map}_{\mathbf{O}}^{\mathrm{h}}(\mathcal{A}, \mathcal{O})_{\omega} \sim \operatorname{Map}_{\mathcal{A}-\mathcal{A}}^{\mathrm{h}}(\mathcal{A}, \mathcal{O}),
$$

where on the right $\mathcal{O}$ is treated as a $\mathcal{A}-\mathcal{A}$-bimodule via $\omega$.

1.10 The second application of Theorem 1.7 This is more peculiar. For any two objects $X, Y$ of $\mathbf{S}$, the graded cartesian product $X \odot Y$ is the graded space defined by

$$
(X \odot Y)_{n}=\coprod_{i+j=n} X_{i} \times Y_{j}
$$

The unit for $\odot$ is the graded space $e_{\odot}$ which is empty except for a single point at level 0; under the pairing $\odot$ the category $\mathbf{S}$ becomes a symmetric monoidal category. It is easy to see that a graded space $X$ is a $\odot$-monoid $(X \odot X \rightarrow X)$ if and only if it is a left $\mathcal{A}$-module $(\mathcal{A} \circ X \rightarrow X)$. But more is true. For any three graded spaces $X$, $Y, Z$ there is a natural distributive isomorphism

$$
(X \odot Y) \circ Z \cong(X \circ Z) \odot(Y \circ Z) .
$$


This guarantees that if $X$ and $Y$ are right modules over an operad $\mathcal{P}$, then $X \odot Y$ is also naturally a right module over $\mathcal{P}$; indeed, $\odot$ provides a symmetric monoidal structure on the category $\mathbf{S}_{\mathcal{P}}$ of right $\mathcal{P}$-modules, such that the monoids in $\left(\mathbf{S}_{\mathcal{P}}, \odot, e_{\odot}\right)$ are exactly the $\mathcal{A}-\mathcal{P}$-bimodules in $\left(\mathbf{S}, \circ, e_{\circ}\right)$.

In our second application of Theorem 1.7, we take $\mathcal{P}=\mathcal{A}$ in the above remarks and we let $\left(\mathbf{C}, \diamond, e_{\diamond}\right)$ be $\left(\mathbf{S}_{\mathcal{A}}, \odot, e_{\odot}\right)$. Let $A$ denote the associative operad $\mathcal{A}$ with an emphasis on its role as a monoid in $\mathbf{S}_{\mathcal{A}}$. Theorem 1.7 then translates to this.

1.12 Theorem (Section 7) Suppose that $\alpha: \mathcal{A} \rightarrow X$ is a map of $\mathcal{A}-\mathcal{A}$-bimodules, and that $X_{0} \sim *$. Then there is an equivalence

$$
\Omega \operatorname{Map}_{\mathcal{A}-\mathcal{A}}^{\mathrm{h}}(\mathcal{A}, X)_{\alpha} \sim \operatorname{Map}_{A-A, \mathcal{A}}^{\mathrm{h}}(A, X) .
$$

The mapping space on the right is computed in the category of $A-A$-bimodules with respect to $\odot$ in a setting in which all of the graded spaces involved are right $\mathcal{A}$-modules with respect to $\circ$ ! Fortunately, this category is a lot less complicated than it looks; in fact it is exactly the category of cosimplicial spaces. We thank the referee for highlighting this. Theorem 1.1 now follows easily from Theorems 1.12 and 1.9.

1.13 Some additional comments Axiom $E$ is a disguised form of another assumption. In fortunate cases, enhancing a distinguished right $S$-module $X_{S}$ to an $R-S-$ bimodule amounts to giving a map from $R$ to an enriched endomorphism object End $^{+}\left(X_{S}\right)$, but Axiom E suggests that such an enhancement amounts to a map $R \rightarrow S$ of monoidal objects. In spirit, then, Axiom $\mathrm{E}$ is the assumption that $S \sim \operatorname{End}^{+}\left(X_{S}\right)$ or more explicitly, given that $X_{S}$ is distinguished, that $S$ is equivalent to the enriched endomorphism object of $S$ itself as a right $S$-module. One feature of Axiom E is that it avoids any direct consideration of what such an enriched endomorphism object might be.

In practice we prove a delooped version of Theorem 1.7. Let $\mathcal{M}^{\mathrm{d}}(R, S)$ denote the moduli space of distinguished $R-S$-bimodules; this is the nerve of the category whose objects are distinguished $R-S$-bimodules and whose morphisms are the equivalences between them. This can be identified (Section 2.1) as

$$
\mathcal{M}^{\mathrm{d}}(R, S) \sim \coprod_{\{X\}} B \operatorname{Aut}_{R-S}^{\mathrm{h}}(X),
$$

where $\{X\}$ runs over equivalence classes of distinguished $R-S$-bimodules, and $\operatorname{Aut}_{R-S}^{\mathrm{h}}(X)$ is the space of derived self-equivalences of $X$. 
1.14 Theorem (cf Theorem 3.9) In the situation of Theorem 1.7 there is an equivalence

$$
\operatorname{Map}_{\mathbf{M}}^{\mathrm{h}}(R, S) \sim \mathcal{M}^{\mathrm{d}}(R, S) .
$$

There is another way to express Theorem 1.14. Let $\mathcal{M}^{\mathrm{pd}}(R, S)$ denote the moduli space of all potentially distinguished $R-S$-bimodules, ie, bimodules which are (abstractly) equivalent to $S$ as right $S$-modules, so that in particular $\mathcal{M}^{\mathrm{pd}}\left(e_{\diamond}, S\right)$ is the moduli space of all potentially distinguished right $S$-modules. This last space is weakly equivalent to $B \operatorname{Aut}_{S}^{\mathrm{h}}(S)$, where the notation indicates that $\mathrm{Aut}^{\mathrm{h}}$ is computed in the category of right $S$-modules. Unpacking the basepoint from Theorem 1.14 (cf Theorem 3.10) reveals that there is a fibration sequence

$$
\operatorname{Map}_{\mathbf{M}}^{\mathrm{h}}(R, S) \rightarrow \mathcal{M}^{\mathrm{pd}}(R, S) \rightarrow \mathcal{M}^{\mathrm{pd}}\left(e_{\diamond}, S\right) .
$$

Here are some contexts $\left(\mathbf{C}, \diamond, e_{\diamond}\right)$ in which $(1.15)$ comes up.

Simplicial monoids Here $\mathbf{C}$ is the category $\mathbf{S p}$ and $\diamond$ is cartesian product. A monoidal object is a simplicial monoid. If $G$ and $H$ are two simplicial monoids which happen to be simplicial groups, then (1.15) can be identified with the fibration sequence

$$
\operatorname{Map}_{*}(B G, B H) \rightarrow \operatorname{Map}(B G, B H) \rightarrow B H .
$$

Operads Here $\mathbf{C}$ is the category of symmetric sequences in $\mathbf{S p}, \diamond$ is the appropriate analog of the composition product, and the monoidal objects are $\Sigma$-operads. Let $\mathcal{P}$ and $\mathcal{Q}$ be two $\Sigma$-operads. Under the additional assumption that $\mathcal{Q}$ is an endomorphism operad, the fibration $(1.15)$ for $\operatorname{Map}_{\mathbf{M}}^{\mathrm{h}}(\mathcal{P}, \mathcal{Q})$ appears as [21, 1.1.5].

Ring spectra Here $\mathbf{C}$ is the category of spectra, $\diamond$ is smash product and the monoidal objects are ring spectra. Let $R$ and $S$ be two ring spectra. Lazarev [15] gives a calculation of $\Omega \operatorname{Map}_{\mathbf{M}}^{\mathrm{h}}(R, S)$ very similar to Theorem 1.7. In this case (1.15) gives a fibration sequence

$$
\operatorname{Map}_{\mathbf{M}}^{\mathrm{h}}(R, S) \rightarrow \mathcal{M}^{\mathrm{pd}}(R, S) \rightarrow B S^{\times},
$$

where $S^{\times}$is the group-like simplicial monoid of units in $S$.

1.16 Notation and terminology The word equivalence usually refers to equivalence in an ambient model category; to avoid certain ambiguities, we sometimes use weak equivalence or weak homotopy equivalence to refer to an equivalence in the usual model category of simplicial sets. We sometimes elide the distinction between a category and its nerve, so that a functor is described as a weak equivalence if it induces a weak equivalence on nerves. Adjoint functors are always weak equivalences; more 
generally, a functor $F: \mathbf{C} \rightarrow \mathbf{D}$ is a weak equivalence if there is a functor $G: \mathbf{D} \rightarrow \mathbf{C}$ such that the composites $F G$ and $G F$ are connected to the identity functor by zigzags of natural transformations. If $\mathbf{C}$ is a category with some notion of equivalence, the moduli category $\mathcal{M}(\mathbf{C})$ is the category of equivalences in $\mathbf{C}$. The moduli space of $\mathbf{C}$ (denoted identically) is the nerve of the moduli category.

If $F: \mathbf{C} \rightarrow \mathbf{D}$ is a functor and $d$ is an object of $\mathbf{D}, F \searrow d$ denotes the over category (comma category) of $F$ with respect to $d$. This is the category whose objects are pairs $(c, g)$ where $c \in \mathbf{C}$ and $g$ is a map $F(c) \rightarrow d$ in $\mathbf{D}$; a morphism $(c, g) \rightarrow\left(c^{\prime}, g^{\prime}\right)$ is a map $c \rightarrow c^{\prime}$ in $\mathbf{C}$ rendering the appropriate diagram commutative. The corresponding under category is denoted $d \searrow F$. If $F$ is the identity functor on $\mathbf{D}$, these categories are denoted $\mathbf{D} \searrow d$ and $d \searrow \mathbf{D}$.

Suppose that $F: \mathbf{C} \rightarrow \mathbf{D}$ is a functor such that for every $h: d \rightarrow d^{\prime}$ in $\mathbf{D}$ the map $F \searrow d \rightarrow F \searrow d^{\prime}$ induced by composition with $h$ is a weak homotopy equivalence; in this circumstance Quillen's Theorem B guarantees that for any $d \in \mathbf{D}$ the homotopy fiber of (the nerve of) $F$ over the vertex of $\mathbf{D}$ represented by $d$ is naturally weakly homotopy equivalent to $F \searrow d$. A similar result holds with over categories replaced by under categories.

Our symbol for coproduct is usually $\sqcup$, or $\sqcup^{\mathbf{D}}$ if the ambient category is specified; in Sections 4 and 6 the symbol $\cup$ is used for the coproduct of graded spaces. We refer to the dimension of a simplex in a space, the level or grade of a constituent of a graded space and the simplicial degree of a constituent of a (co)simplicial (graded) space.

1.17 Remark In this paper we work only with non- $\Sigma$ operads, also called planar operads. Many of our results apply to $\Sigma$-operads (symmetric operads) or even to multicategories, but we decided to leave this generality for later.

Organization of the paper Section 2 sets up some model category machinery, which is used in Section 3 to give a proof of Theorem 1.7. Section 4 moves to a more concrete consideration of model structures on operads of spaces and proves the crucial result that the coproduct functor preserves equivalences. Section 5 describes the Hochschild resolution of an operad $\mathcal{P}$ as a bimodule over itself and shows that applying an enveloping construction to the Hochschild resolution leads to a resolution of $\mathcal{P}$ as an operad; Section 6 uses this last resolution to give a proof of Theorem 1.9. The final two sections transpose the earlier results to the context of classic monoids in the category of graded spaces, and go on to deduce Theorems 1.12 and 1.1.

For our approach to the homotopy theory of operads we are deeply indebted to the results of Rezk [22]. The first author was partially supported by NSF grant DMS 0354787. 


\section{Model categories}

In this section we develop basic properties of model categories which we will need later on; Hovey [13] and Goerss and Jardine [8] are two background references. The underlying definition of model category is from [13, 1.1.4]; in particular, a model category is closed in the sense of Quillen [20], has functorial factorizations [13, 1.1.1], and has all small limits and colimits.

2.1 Some generalities Suppose that $\mathbf{D}$ is a model category. The symbol $\sim$ marks equivalences in D. A (co)fibration is acyclic if it is also an equivalence. A cofibrant replacement for an object $X$ is an equivalence $X^{\mathrm{c}} \stackrel{\sim}{\rightarrow} X$ with cofibrant domain, usually obtained (functorially) by factoring the map from the initial object to $X$ as a cofibration followed by an acyclic fibration. A fibrant replacement $X \stackrel{\sim}{\rightarrow} X^{\mathrm{f}}$ is constructed similarly.

Quillen pairs and Quillen equivalences An adjoint pair $\lambda$ : D $\leftrightarrow \mathbf{E}: \rho$ of functors between model categories is a Quillen pair $[13,1.3 .1]$ if $\lambda$ preserves cofibrations and acyclic cofibrations (equivalently, $\rho$ preserves fibrations and acyclic fibrations). In this case, $\lambda$ preserves equivalences between cofibrant objects and $\rho$ preserves equivalences between fibrant objects. The pair $(\lambda, \rho)$ forms a Quillen equivalence $[13,1.3 .3]$ if for all cofibrant $X \in \mathbf{D}$ and fibrant $Y \in \mathbf{E}$, a map $\lambda X \rightarrow Y$ is an equivalence in $\mathbf{E}$ if and only if the adjoint $X \rightarrow \rho Y$ is an equivalence in $\mathbf{D}$. Let $\mathbf{D}^{\mathrm{c}}, \mathbf{D}^{\mathrm{f}}$, etc., denote appropriate full subcategories of cofibrant or fibrant objects. Given a Quillen pair $(\lambda, \rho)$ there is an induced diagram of moduli spaces

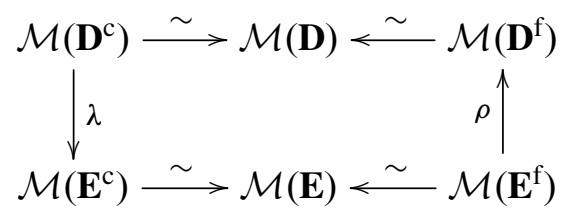

in which (by functorial fibrant/cofibrant replacement constructions) the indicated arrows are homotopy equivalences with explicit homotopy inverses. It is not hard to see that if $(\lambda, \rho)$ is a Quillen equivalence, then both vertical arrows are weak homotopy equivalences, and that these arrows are homotopy inverse to one another in an appropriate sense; see Dwyer et al $[4,17.5]$. In particular, if $\rho$ preserves all equivalences, then the map $\mathcal{M}(\mathbf{E}) \rightarrow \mathcal{M}(\mathbf{D})$ induced by $\rho$ is a weak homotopy equivalence.

Derived mapping spaces If $X$ and $Y$ are objects of $\mathbf{D}, \operatorname{Map}_{\mathbf{D}}^{\mathrm{h}}(X, Y)=\operatorname{Map}^{\mathrm{h}}(X, Y)$ denotes the simplicial set of derived maps $X \rightarrow Y$; technically, this is defined in terms of the hammock localization (see Dwyer and Kan [5]), and it depends only on the equivalences in D. If $\mathbf{D}$ is a simplicial model category [8, II.3], then $\operatorname{Map}^{\mathrm{h}}(X, Y)$ is 


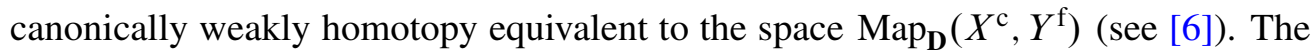
bifunctor $\operatorname{Map}^{\mathrm{h}}(-,-)$ converts an equivalence in either variable into a weak homotopy equivalence. By $[5,1.1]$ the space $\operatorname{Map}^{\mathrm{h}}(X, Y)$ is canonically weakly equivalent to the nerve of the category depicted pictorially as follows

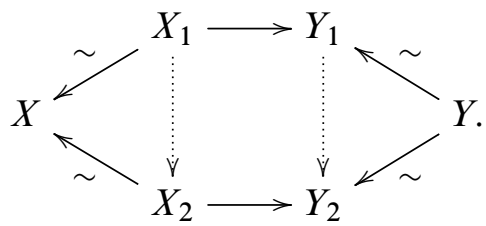

In this convention, zigzagging across the top gives one object of this category, zigzagging across the bottom another, and the entire commutative diagram with the dotted arrows drawn in represents a morphism from the top object to the bottom one. Dugger has shown [3] that if $X$ is cofibrant, $\operatorname{Map}^{\mathrm{h}}(X, Y)$ is equivalent to the nerve of the less complicated category

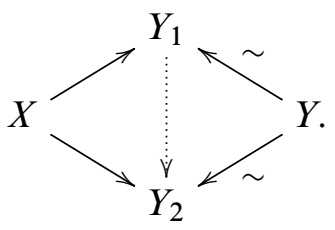

Remark Note that this is a certain subcategory of the category of objects under $X \sqcup Y$. If $\mathbf{D}$ is a category of monoids in some underlying monoidal category (Section 1.5), then this is the category of distinguished monoids under $X \sqcup Y$. Any such distinguished monoid has an underlying distinguished $X-Y$ bimodule, and in this paper we exploit the fact that in fortunate circumstances passing to distinguished bimodules does not alter the homotopy type of the categorical nerves (Theorem 3.9).

2.5 Left proper model categories We need a slight generalization of Dugger's result. The model category $\mathbf{D}$ is left proper if the pushout of an equivalence along a cofibration is again an equivalence [8, II.8.P2]. In [22, 2.7], Rezk observes that $\mathbf{D}$ is left proper if and only if for any equivalence $Z \rightarrow Z^{\prime}$ in $\mathbf{D}$ the restriction functor $\rho: Z^{\prime} \searrow \mathbf{D} \rightarrow Z \searrow \mathbf{D}$ is the right adjoint of a Quillen equivalence. Since $\rho$ preserves all weak equivalences, it follows that $\rho$ induces a weak equivalence $\mathcal{M}\left(Z^{\prime} \searrow \mathbf{D}\right) \stackrel{\sim}{\rightarrow} \mathcal{M}(Z \searrow \mathbf{D})$ (see (2.2) ff).

2.6 Proposition Suppose that $\mathbf{D}$ is left proper, and that $X$ and $Y$ are objects of $\mathbf{D}$ such that $X^{\mathrm{c}} \sqcup Y \rightarrow X \sqcup Y$ is an equivalence. Then the nerve of the category (2.4) has the weak homotopy type of $\operatorname{Map}^{\mathrm{h}}(X, Y)$. 
Proof Let $Z=X^{\mathrm{c}} \sqcup Y$ and $Z^{\prime}=X \sqcup Y$. The nerve of (2.4) is a union of components of $\mathcal{M}\left(Z^{\prime} \searrow \mathbf{D}\right)$, and according to Dugger, the corresponding union of components of $\mathcal{M}(Z \searrow \mathbf{D})$ computes $\operatorname{Map}^{\mathrm{h}}(X, Y)$. If $f: Z \rightarrow Z^{\prime}$ is an equivalence, it follows from Rezk's observation above that the map of moduli spaces induced by restriction over $f$ is a weak equivalence. Since this map preserves the appropriate components, the conclusion follows.

2.7 Remark Let $\operatorname{Aut}_{\mathbf{D}}^{\mathrm{h}}(X)=\operatorname{Aut}^{\mathrm{h}}(X)$ denote the union of those components of $\operatorname{Maph}^{\mathrm{h}}(X, X)$ which are invertible up to homotopy. There is a natural weak homotopy equivalence

$$
B \operatorname{Aut}^{\mathrm{h}}(X) \sim \mathcal{M}(\mathbf{D})_{X},
$$

where $\mathcal{M}(\mathbf{D})_{X}$ is the component of the moduli space $\mathcal{M}(\mathbf{D})$ (Section 1.16) corresponding to $X$. This is proved by stringing together results from $[7,5.5 ; 5,2.2$; 6, 4.6(ii)].

2.9 Quillen pairs and homotopy fibres of moduli spaces Suppose that $(\lambda, \rho)$ is a Quillen pair as above, such that $\rho$ preserves all equivalences and thus induces a map $\mathcal{M}(\rho): \mathcal{M}(\mathbf{E}) \rightarrow \mathcal{M}(\mathbf{D})$. We are interested in showing that the homotopy fibre of $\mathcal{M}(\rho)$ over $A \in \mathcal{M}(\mathbf{D})$ is often given by the nerve of the under category $A \searrow \mathcal{M}(\rho)$.

2.10 Proposition Suppose that $\lambda$ : $\mathbf{D} \leftrightarrow \mathbf{E}: \rho$ is a Quillen pair such that $\rho$ preserves all equivalences. Then the homotopy fibre of $\mathcal{M}(\rho)$ over $A \in \mathcal{M}(\mathbf{D})$ is given by $A \searrow \mathcal{M}(\rho)$ if either

(1) $A$ is cofibrant, or

(2) $\mathbf{E}$ is left proper and $\lambda\left(A^{\mathrm{c}}\right) \rightarrow \lambda(A)$ is an equivalence.

Proof Consider the following two categories:

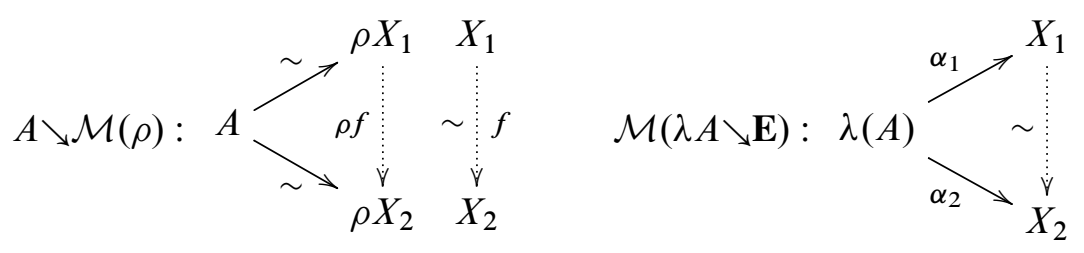

The fact that $\rho$ preserves equivalences implies that $A \searrow \mathcal{M}(\rho)$ is isomorphic to the union of the components of $\mathcal{M}(\lambda A \searrow \mathbf{E})$ containing maps $\alpha: \lambda A \rightarrow X$ whose adjoint $\alpha^{b}: A \rightarrow \rho X$ is an equivalence. Two conclusions follow. First, if assumption (2) holds the natural map $A \searrow \mathcal{M}(\rho) \rightarrow A^{\mathrm{c}} \searrow \mathcal{M}(\rho)$ is a weak homotopy equivalence (Section 2.5), 
and hence without loss of generality we can assume that (1) holds and $A$ is cofibrant. Secondly, if $A \rightarrow A^{\prime}$ is an equivalence between cofibrant objects of $\mathbf{D}$, the natural map $A^{\prime} \searrow \mathcal{M}(\rho) \rightarrow A \searrow \mathcal{M}(\rho)$ is a weak homotopy equivalence [22, 2.5].

We now perturb the problem into one which can be solved by combining this last observation with Quillen's Theorem B (Section 1.16). Let $\mathbf{D}^{\mathbf{c}} \subset \mathbf{D}$ be the subcategory of cofibrant objects, and consider the two categories described pictorially below (all arrows are equivalences).

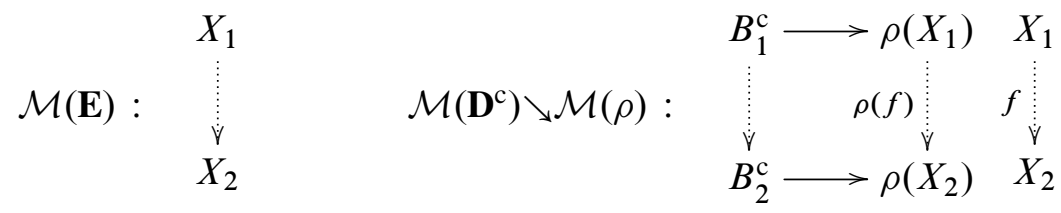

The category $\mathcal{M}\left(\mathbf{D}^{\mathrm{c}}\right) \backslash \mathcal{M}(\rho)$ is a path space construction in which an object consists of object $X_{1} \in \mathbf{E}$, an object $B_{1}^{\mathrm{c}} \in \mathbf{D}^{\mathrm{c}}$, and an equivalence $B_{1}^{\mathrm{c}} \rightarrow \rho\left(X_{1}\right)$; a morphism consists of equivalences $X_{1} \rightarrow X_{2}$ and $B_{1}^{\mathrm{c}} \rightarrow B_{2}^{\mathrm{c}}$ making the indicated diagram commute. Let $u: \mathbf{D}^{\mathrm{c}} \rightarrow \mathbf{D}$ denote the inclusion. There is a diagram of functors

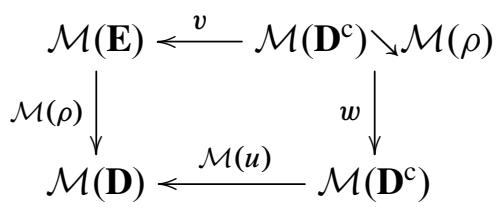

where $v$ picks out $X_{1}$ and $w$ picks out $B_{1}^{c}$; the square commutes up to an explicit natural transformation. The functors $\mathcal{M}(u)$ and $v$ are weak homotopy equivalences, since there are oppositely oriented functors (given by choosing functorial cofibrant replacements) such that composites are connected to appropriate identity functors by natural transformations. If $A$ is cofibrant, it is easy to construct a functor $A \searrow \mathcal{M}(\rho) \rightarrow$ $A \searrow w$ (natural in $A$ ) which is a weak homotopy equivalence. As above, this implies that any equivalence $A \rightarrow A^{\prime}$ in $\mathbf{D}^{\mathrm{c}}$ induces a weak homotopy equivalence $A^{\prime} \searrow w \rightarrow A \searrow w$. By Quillen's Theorem B, the homotopy fibre of $w$ over $A$, or equivalently the homotopy fibre of $\mathcal{M}(\rho)$ over $A$, is weakly homotopy equivalent to $A \searrow w \sim A \searrow \mathcal{M}(\rho)$.

2.11 Quillen pairs and mapping spaces We will also need that Quillen pairs are topologically adjoint with respect to model-category theoretic mapping spaces. A version of this up to homotopy is included in [13, 5.6.2] (cf $[12,17.4 .15]$ ), but we prefer a slightly more rigid formulation. As usual, $A^{\mathrm{c}} \stackrel{\sim}{\rightarrow} A$ and $Y \stackrel{\sim}{\rightarrow} Y^{\mathrm{f}}$ are cofibrant and fibrant replacements. 
2.12 Theorem Suppose that $\lambda: \mathbf{D} \leftrightarrow \mathbf{E}: \rho$ is a Quillen pair, and that $A$ and $Y$ are objects of $\mathbf{D}$ and $\mathbf{E}$ respectively. Then there is a natural weak homotopy equivalence

$$
\operatorname{Map}_{\mathbf{D}}^{\mathrm{h}}\left(A, \rho\left(Y^{\mathrm{f}}\right)\right) \sim \operatorname{Map}_{\mathbf{E}}^{\mathrm{h}}\left(\lambda\left(A^{\mathrm{c}}\right), Y\right) .
$$

Proof We can assume without loss of generality that $A$ is cofibrant and $Y$ is fibrant. Consider the category $\mathbf{Z}$ described by the diagram

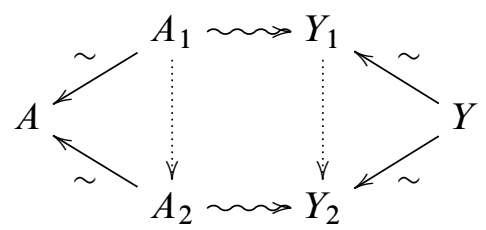

where an undulating arrow $A_{i} \leadsto Y_{i}$ represents (equivalently) a map $A_{i} \rightarrow \rho\left(Y_{i}\right)$ in $\mathbf{D}$ or a map $\lambda\left(A_{i}\right) \rightarrow Y_{i}$ in $\mathbf{E}$. We will show that $\mathbf{Z}$ is weakly equivalent to $\operatorname{Map}^{\mathrm{h}}(\lambda A, Y)$; a dual argument shows that it is weakly equivalent to $\operatorname{Map}^{\mathrm{h}}(A, \rho Y)$.

Let $\mathbf{D}^{\mathrm{c}}$ be the category of cofibrant objects in $\mathbf{D}$. A functorial factorization argument shows that $\mathbf{Z}$ is weakly homotopy equivalent to the full subcategory $\mathbf{Z}^{\mathrm{c}}$ consisting of zigzags with $A_{1} \in \mathbf{D}^{\mathrm{c}}$. Picking off $A_{1} \stackrel{\sim}{\rightarrow} A$ gives a functor $F: \mathbf{Z}^{\mathrm{c}} \rightarrow \mathcal{M}\left(\mathbf{D}^{\mathrm{c}}\right) \searrow A$. Given an object $U=\left\langle A^{\prime} \stackrel{\sim}{\rightarrow} A\right\rangle$ of $\mathcal{M}\left(\mathbf{D}^{\mathrm{c}}\right) \searrow A$, the over category $U \searrow F$ has objects consisting of diagrams of the form

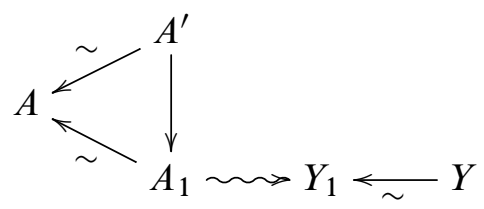

This is an object of $U \searrow F$; in this category, $A, A^{\prime}$ and $Y$ are fixed, but $A_{1}$ and $Y_{1}$ are allowed to vary. This category is homotopy equivalent to the subcategory consisting of objects in which $A^{\prime} \rightarrow A_{1}$ is the identity map; by (2.4), this subcategory has the weak homotopy type of $\operatorname{Map}_{\mathbf{E}}^{\mathrm{h}}\left(\lambda\left(A^{\prime}\right), Y\right)$ and so in particular the weak homotopy type is independent of the choice $U$ of object. By Quillen's Theorem $\mathrm{B}, \operatorname{Map}_{\mathbf{E}}^{\mathrm{h}}(\lambda A, Y)$ is equivalent to the homotopy fibre of $F$. Since the target $\mathcal{M}\left(\mathbf{D}^{\mathrm{c}}\right) \searrow A$ of $F$ is contractible (this category has a terminal object), it follows that $\operatorname{Map}_{\mathbf{E}}^{\mathrm{h}}(\lambda A, Y)$ is weakly equivalent to $\mathbf{Z}$.

\section{Monoidal categories and the proof of Theorem 1.7}

In this section we work out the machinery sketched in Section 1.5. The triple $\left(\mathbf{C}, \diamond, e_{\diamond}\right)$ will be a monoidal category in the sense of [16, VII.1], and $\mathbf{M}$ the category of monoids 
in $\mathbf{C}$ [16, VII.3]. For the rest of this section $R$ and $S$ are two fixed objects of $\mathbf{M}$. We use the following notation:

- $\mathbf{C}_{S}=$ the category of right $S$-modules,

- $\mathbf{C}_{R, S}=$ the category of $R-S$-bimodules, and

- $\mathbf{M}_{R, S}=$ the category of monoidal objects under $R$ and $S$.

A point for an object $X \in \mathbf{C}$ is defined to be a $\mathbf{C}$-map $e_{\diamond} \rightarrow X$; a map between pointed objects is required to respect the points. A superscript $e$ indicates a category of pointed objects, so that for instance,

- $\mathrm{C}_{R, S}^{e}=$ the category of pointed $R-S$-bimodules.

Note that the category of pointed $R-S$-bimodules is isomorphic to the category of $R-S$-bimodules under $R \diamond S$. We now introduce the axioms under which Theorem 1.7 holds.

3.1 Axiom I The categories $\mathbf{C}, \mathbf{M}, \mathbf{C}_{S}$, and $\mathbf{C}_{R, S}$ possess compatible (see below) model category structures.

The term compatible means that a map in one of these categories is an equivalence or a fibration if and only if the underlying map in $\mathbf{C}$ has the same property. For general reasons, the above model category structures on $\mathbf{M}$ and on $\mathbf{C}_{R, S}$ extend to compatible model structures on $\mathbf{M}_{R, S}$ and $\mathbf{C}_{R, S}^{e}[13,1.1 .8 \mathrm{ff}]$.

There is a forgetful functor $\downarrow: \mathbf{M}_{R, S} \rightarrow \mathbf{C}_{R, S}^{e}$, with the point for $\downarrow(T)$ provided by the unit map $e_{\diamond} \rightarrow T$.

3.2 Axiom II The functor $\pitchfork: \mathbf{M}_{R, S} \rightarrow \mathbf{C}_{R, S}^{e}$ has a left adjoint $E$.

3.3 Remark The compatibility condition in Axiom I guarantees that $\downarrow$ preserves fibrations and equivalences, and it follows that $(E, \downarrow)$ forms a Quillen pair. We will let $E^{\mathrm{h}}$ denote the left derived functor of $E$, which is given by $E^{\mathrm{h}}(X)=E\left(X^{\mathrm{c}}\right)$, where $X^{\mathrm{c}} \rightarrow X$ is a functorial cofibrant replacement in $\mathbf{C}_{R, S}^{e}$. See [13, 1.3.2], but note that for us the codomain of $E^{\mathrm{h}}$ is $\mathbf{M}_{R, S}$, not the homotopy category of $\mathbf{M}_{R, S}$.

Given $X \in \mathbf{C}_{S}^{e}$, there is a right $S$-module map

$$
S \cong e_{\diamond} \diamond S \rightarrow X
$$

derived from the point in $X$. The object $X$ is distinguished if this map is an equivalence. An object of $\mathbf{C}_{R, S}^{e}$ is distinguished if it is distinguished as a pointed right $S$-module. Similarly, an object $T \in \mathbf{M}_{R, S}$ is distinguished if the structure map $S \rightarrow T$ is an equivalence. 
3.4 Axiom III The derived functor $E^{\mathrm{h}}: \mathbf{C}_{R, S}^{e} \rightarrow \mathbf{M}_{R, S}$ preserves distinguished objects.

The remaining axioms are more technical and have the flavor of nondegeneracy assumptions. Let $R^{\mathrm{c}} \rightarrow R$ be a cofibrant replacement for $R$ in $\mathbf{M}$.

3.5 Axiom IV One of the following two conditions holds:

(1) $R$ itself is cofibrant as an object of $\mathbf{M}$, or

(2) $\mathbf{M}$ is left proper (Section 2.5), and the map $R^{\mathrm{c}} \sqcup^{\mathbf{M}} S \rightarrow R \sqcup^{\mathbf{M}} S$ is an equivalence.

In the next statement, $S^{\mathrm{c}} \rightarrow S$ is a cofibrant replacement in $\mathbf{C}_{S}$.

3.6 Axiom $\mathbf{V}$ One of the following two conditions holds:

(1) $S$ itself is cofibrant as an object of $\mathbf{C}_{S}$, or

(2) $\mathbf{C}_{R, S}$ is left proper, and the map $R \diamond S^{\mathrm{c}} \rightarrow R \diamond S$ is an equivalence.

3.7 Remark It is very tempting to assume that $S$ is necessarily cofibrant as an object of $\mathbf{C}_{S}$, since $S$ is the free right $S$-module on one generator. But the notion of "one generator" here is tricky: it is more accurate to say that $S$ is the free right $S$ module on the object $e_{\diamond}$ of $\mathbf{C}$. The real issue is whether $e_{\diamond}$ is cofibrant in $\mathbf{C}$.

If $M$ is an $R$-R-bimodule and $\omega: R \rightarrow S$ is a map of monoidal objects, write $M \diamond_{R}^{\omega} S$ for the coequalizer of the two maps $M \diamond R \diamond S \rightarrow M \diamond S$ obtained by pairing the central $R$ either with $M$ or (via $\omega$ ) with $S$; the coequalizer is to be computed in the category $\mathbf{C}_{R, S}$. The functor $M \mapsto M \diamond_{R}^{\omega} S$ is left adjoint to the functor $\mathbf{C}_{R, S} \rightarrow \mathbf{C}_{R, R}$ induced by composition with $\omega$.

3.8 Axiom VI Suppose that $\omega: R \rightarrow S$ is a map of monoidal objects, $e_{\diamond}^{\mathrm{c}} \rightarrow e_{\diamond}$ is a cofibrant replacement for $e_{\diamond}$ in $\mathbf{C}$, and $R^{\mathrm{c}} \rightarrow R$ is a cofibrant replacement for $R$ in $\mathbf{C}_{R, R}$. Then the following two conditions hold.

(1) $e_{\diamond}^{\mathrm{c}} \diamond S \rightarrow e_{\diamond} \diamond S \cong S$ is an equivalence (in $\mathbf{C}_{S}$ ), and

(2) $R^{\mathrm{c}} \diamond_{R}^{\omega} S \rightarrow R \diamond_{R}^{\omega} S \cong S$ is an equivalence (in $\mathbf{C}_{R, S}$ ).

We first prove Theorem 1.14, which relates maps between monoid objects to moduli spaces of pointed bimodules. Recall from Section 2.1 that $\operatorname{Map}_{\mathbf{M}}^{\mathrm{h}}(R, S)$ denotes the space of maps $R \rightarrow S$ provided by the model category structure on $\mathbf{M}$. Let $\mathbf{C}_{R, S}^{\mathrm{d}}$ be the subcategory of $\mathbf{C}_{R, S}^{e}$ given by the distinguished objects; all morphisms in this category are equivalences, so $\mathcal{M}\left(\mathbf{C}_{R, S}^{\mathrm{d}}\right)=\mathbf{C}_{R, S}^{\mathrm{d}}$ is just the nerve. 
3.9 Theorem If Axioms I-IV hold, then there is a natural weak equivalence of spaces

$$
\operatorname{Map}_{\mathbf{M}}^{\mathrm{h}}(R, S) \sim \mathcal{M}\left(\mathbf{C}_{R, S}^{\mathrm{d}}\right) .
$$

Proof of Theorem 3.9 Combining Axiom IV with Section 2.5 shows there is a natural weak equivalence $\operatorname{Map}_{\mathbf{M}}^{\mathrm{h}}(R, S) \sim \mathcal{M}\left(\mathbf{M}_{R, S}^{\mathrm{d}}\right)$, where $\mathbf{M}_{R, S}^{\mathrm{d}}$ is the category of all distinguished objects in $\mathbf{M}_{R, S}$ and all maps (necessarily equivalences) between them. Recall that for $X \in \mathbf{C}_{R, S}^{e}, E^{\mathrm{h}}(X)$ can be computed as $E\left(X^{\mathrm{c}}\right)$, where $X^{\mathrm{c}} \stackrel{\sim}{\rightarrow} X$ is a functorial cofibrant replacement for $X$. The functor $\downarrow$ restricts to a functor $\mathbf{M}_{R, S}^{\mathrm{d}} \rightarrow \mathbf{C}_{R, S}^{\mathrm{d}}$ and it follows from Axiom III that $E^{\mathrm{h}}$ restricts to a functor $\mathbf{C}_{R, S}^{\mathrm{d}} \rightarrow \mathbf{M}_{R, S}^{\mathrm{d}}$. The arrows

$$
E\left(\left(\llcorner T)^{\mathrm{c}}\right) \rightarrow T \quad \text { and } \quad X \leftarrow X^{\mathrm{c}} \rightarrow \natural\left(E\left(X^{\mathrm{c}}\right)\right)\right.
$$

show that the composites $E \natural$ and $\llbracket E$ of these restricted functors are each connected to the respective identity functor by a chain of natural transformations; it follows $\mathbf{C}_{R, S}^{\mathrm{d}}$ and $\mathbf{M}_{R, S}^{\mathrm{d}}$ have weakly equivalent nerves.

Call a right $S$ module potentially distinguished if it is (abstractly) equivalent to $S$ itself, and let $\mathbf{C}_{S}^{\mathrm{pd}}$ denote the full subcategory of $\mathbf{C}_{S}$ containing the objects which are potentially distinguished. Similarly, let $\mathbf{C}_{R, S}^{\text {pd }}$ be the full subcategory of $\mathbf{C}_{R, S}$ containing bimodules which are potentially distinguished as right $S$-modules.

The next statement unbundles the basepoint from Theorem 3.9.

3.10 Theorem If Axioms I-V hold, then there is a natural fibration sequence

$$
\operatorname{Map}_{\mathbf{M}}^{\mathrm{h}}(R, S) \rightarrow \mathcal{M}\left(\mathbf{C}_{R, S}^{\mathrm{pd}}\right) \rightarrow \mathcal{M}\left(\mathbf{C}_{S}^{\mathrm{pd}}\right),
$$

where the fibre is to be taken over $S \in \mathbf{C}_{S}^{\mathrm{pd}}$.

Proof Consider the adjoint functors $\lambda$ : $\mathbf{C}_{S} \leftrightarrow \mathbf{C}_{R, S}: \rho$, where $\rho$ forgets the left $R-$ structure and $\lambda(X)=R \diamond X$. As in Remark 3.3, $(\lambda, \rho)$ forms a Quillen pair. By Axiom V and Proposition 2.10, the homotopy fibre of $\mathcal{M}(\rho)$ over $S$ is naturally weakly homotopy equivalent to $\mathcal{M}\left(\mathbf{C}_{R, S}^{\mathrm{d}}\right)$, and hence, by Theorem 3.9 to $\operatorname{Map}_{\mathbf{M}}^{\mathrm{h}}(R, S)$. The theorem follows from the fact that $\mathcal{M}\left(\mathbf{C}_{S}^{\mathrm{pd}}\right)$ is the component of $\mathcal{M}\left(\mathbf{C}_{S}\right)$ containing $S$, while $\mathcal{M}\left(\mathbf{C}_{R, S}^{\mathrm{pd}}\right)$ is the inverse image of this component in $\mathcal{M}\left(\mathbf{C}_{R, S}\right)$.

Finally, we loop down the fibration sequence from Theorem 3.10 and rewrite the spaces involved. 
3.11 Theorem Suppose that Axioms I-VI hold, and that $\omega: R \rightarrow S$ is a map of monoidal objects. Let $S_{\omega}$ denote $S$ considered via $\omega$ as an $R-R$-bimodule. Then there is a natural fibration sequence

$$
\Omega \operatorname{Map}_{\mathbf{M}}^{\mathrm{h}}(R, S)_{\omega} \rightarrow \operatorname{Map}_{\mathbf{C}_{R, R}}^{\mathrm{h}}\left(R, S_{\omega}\right) \rightarrow \operatorname{Map}_{\mathbf{C}}^{\mathrm{h}}\left(e_{\diamond}, S\right) .
$$

3.12 Remark In this statement, $\Omega \operatorname{Map}_{\mathbf{M}}^{\mathrm{h}}(R, S)_{\omega}$ denotes the loop space taken with $\omega$ as the basepoint, and $R$ is to be treated as an $R-R$-bimodule in the natural way. The right hand map is induced by the unit $e_{\diamond} \rightarrow R$, and the fibre is meant to be taken over the unit $e_{\diamond} \rightarrow S$.

Proof of Theorem 3.11 Looping down the fibration sequence from Theorem 3.10 gives a sequence

$$
\Omega \operatorname{Map}_{\mathbf{M}}^{\mathrm{h}}(R, S)_{\omega} \rightarrow \Omega \mathcal{M}\left(\mathbf{C}_{R, S}^{\mathrm{pd}}\right)_{\omega} \rightarrow \Omega \mathcal{M}\left(\mathbf{C}_{S}^{\mathrm{pd}}\right)_{S} .
$$

We begin by considering the right hand space. By general properties of moduli spaces in (2.8), this loop space is equivalent to the subspace $\operatorname{Aut}_{\mathbf{C}_{S}}^{\mathrm{h}}(S)$ of $\operatorname{Map}_{\mathbf{C}_{S}}^{\mathrm{h}}(S, S)$ consisting of homotopically invertible maps. By Axiom VI(1) and Theorem 2.12 (this last applied to the forgetful functor $\mathbf{C}_{S} \rightarrow \mathbf{C}$ and its left adjoint $\left.-\diamond S\right)$ the space $\operatorname{Map}_{\mathbf{C}_{S}}^{\mathrm{h}}(S, S)$ is weakly equivalent to $\operatorname{Map}_{\mathbf{C}}^{\mathrm{h}}\left(e_{\diamond}, S\right)$.

For similar reasons, the middle space is equivalent to the subspace $\operatorname{Aut}_{\mathbf{C}_{R, S}}^{\mathrm{h}}\left(S_{\omega}\right)$ of $\operatorname{Map}_{\mathbf{C}_{R, S}}^{\mathrm{h}}\left(S_{\omega}, S_{\omega}\right)$ consisting of homotopically invertible maps. By Axiom VI(2) and Theorem 2.12 (this last applied to the restriction functor $\omega^{*}: \mathbf{C}_{R, S} \rightarrow \mathbf{C}_{R, R}$ and its left adjoint $\left.-\diamond_{R}^{\omega} S\right)$, the space $\operatorname{Map}_{\mathbf{C}_{R, S}}^{\mathrm{h}}\left(S_{\omega}, S_{\omega}\right)$ is naturally weakly equivalent to $\operatorname{Map}_{\mathbf{C}_{R, R}}^{\mathrm{h}}\left(R, S_{\omega}\right)$.

All in all, there is a commutative diagram

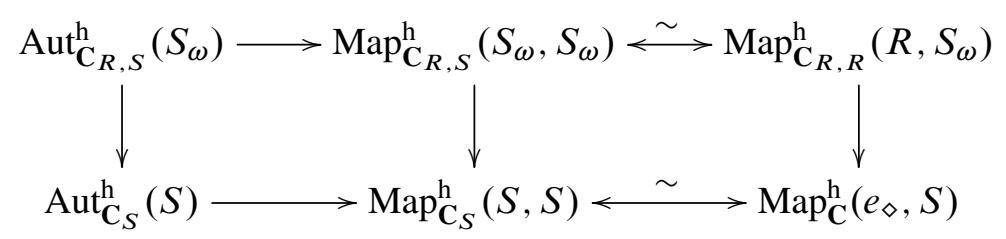

in which the left horizontal arrows are component inclusions. Since a map $S_{\omega} \rightarrow S_{\omega}$ in $\mathbf{C}_{R, S}$ is an equivalence if and only if the underlying map of right $S$-modules is an equivalence, the left hand square is a homotopy fibre square. Consequently, the homotopy fibre over the identity map of $S$ on the left, namely $\Omega \operatorname{Map}_{\mathbf{M}}^{\mathrm{h}}(R, S)_{\omega}$, is equivalent to the homotopy fibre over the image of this identity map on the right. We leave it to the reader to check that this image is the unit $e_{\diamond} \rightarrow S$, and that the right vertical map is as described. 


\section{Model structures, free operads and coproducts of operads}

In this section we develop a few homotopical properties of operads. Deep in the background is the symmetric monoidal category $(\mathbf{S p}, \times, *)$ of simplicial sets with cartesian product, but the ambient monoidal category in this section is the category $\mathbf{S}=$ $\left(\mathbf{S}, \circ, e_{\circ}\right)$ of graded spaces, with the (nonsymmetric) composition monoidal structure from Section 1.2. An operad is a monoid in $\mathbf{S}$. The category $\mathbf{S p}$ has a simplicial model category structure in which the equivalences are the weak homotopy equivalences and the cofibrations are the monomorphisms [8, I.11]. This model structure extends to $\mathbf{S}$ by declaring a map in $\mathbf{S}$ to be an equivalence (resp. cofibration, fibration) if and only if on each level it gives an equivalence (resp. cofibration, fibration) in $\mathbf{S p}$.

Let $\mathbf{S}^{e}$ denote the category of graded spaces furnished with a basepoint at level one, let $\mathbf{O}$ denote the category of operads, and for two chosen operads $\mathcal{P}, \mathcal{Q}$, let $\mathbf{S}_{\mathcal{Q}}$ and $\mathbf{S}_{\mathcal{P}, \mathcal{Q}}$ denote respectively the categories of right $\mathcal{Q}$-modules and $\mathcal{P}-\mathcal{Q}$-bimodules. The forgetful functor $\mathbf{O} \rightarrow \mathbf{S}$ has a left adjoint $\Phi$ (the free operad functor), and the forgetful functor $\mathbf{O} \rightarrow \mathbf{S}^{e}$ obtained by retaining the unit as a basepoint has a left adjoint $\Phi^{e}$. In this section we study model structures on these categories, and prove that $\Phi, \Phi^{e}$, and the coproduct functor on $\mathbf{O}$ are homotopy invariant.

4.1 Proposition [21, Section 3; 22] The categories $\mathbf{O}, \mathbf{S}_{\mathcal{Q}}$, and $\mathbf{S}_{\mathcal{P}, \mathcal{Q}}$ have simplicial model structures compatible (Axiom I) with the model structure on $\mathbf{S}$.

4.2 Remark Rezk produces these model category structures in the setting of $\Sigma-$ operads, but the case of planar operads is a bit simpler; the arguments follow the lines of Goerss and Jardine [8, II.4.1 and II.5.1] (see also Rezk [22, 7.1]). It will be useful later on to understand the cofibrations in these model categories. As in [22, Section 6], a degeneracy object is a simplicial object without face operators. According to [22, Section 6], a map $X \rightarrow Y$ in one of these model categories is a cofibration if it is (a retract of) a monomorphism with the additional property that, as a degeneracy object, $Y$ is isomorphic to the coproduct of $X$ with a free degeneracy object. The notions of free and coproduct here are to be interpreted in the relevant operad or (bi)module setting.

4.3 Proposition The coproduct construction on operads preserves equivalences. The category $\mathbf{O}$ is a left proper model category.

4.4 Proposition The functors $\Phi$ and $\Phi^{e}$ preserve equivalences.

The rest of this section is devoted to proofs of Propositions 4.3 and 4.4; these are routine but involve a substantial amount of notation and bookkeeping. 
4.5 Identities and compositions The identity element of an operad $\mathcal{P}$ is denoted $1_{\mathcal{P}}$. Given a point $a \in \mathcal{P}_{n}$, and $n$ elements $b_{i} \in \mathcal{P}_{k_{i}}(1 \leq i \leq n)$, we use composition notation $a\left(b_{1}, \ldots, b_{n}\right)$ to denote the image of a tuple $\left(a, b_{1}, \ldots, b_{n}\right)$ under the operad structure map.

4.6 Trees and free operads We first describe the set of isomorphism classes of (planar rooted) trees. Each tree $t$ has a height $\chi(t)$, a leaf count $\lambda(t)$, and a branching count $\beta(t)$. The set of trees is defined recursively on height by declaring that there is one tree of height 0 , called the trivial tree and denoted $e$. Its leaf count is 1 , and its branching count is 0 . A tree of height $k>0$ is then a tuple $\left[n ; t_{1}, \ldots, t_{n}\right]$, where $n>0$ is an integer, each $t_{i}$ is a tree of height less than $k$, and at least one $t_{i}$ has height $k-1$. The numerical invariants of a nontrivial tree are given inductively by

$$
\begin{aligned}
& \chi\left[n ; t_{1}, \ldots, t_{n}\right]=1+\max \left\{\chi\left(t_{i}\right)\right\} \text { height, } \\
& \lambda\left[n ; t_{1}, \ldots, t_{n}\right]=\sum \lambda\left(t_{i}\right) \quad \text { leaf count, } \\
& \beta\left[n ; t_{1}, \ldots, t_{n}\right]=1+\sum \beta\left(t_{i}\right) \quad \text { branching count. }
\end{aligned}
$$

Here are some examples:

○

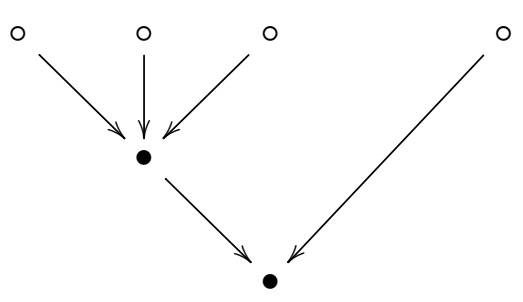

On the left is $e$ : one leaf resting on the ground, no branching. The middle tree is $[3 ; e, e, e]$ : height one, three leaves, one branching. The tree on the right is $[2 ;[3 ; e, e, e], e]$ : height 2,4 leaves, 2 branchings. Trees of branching at most 1 , such as the trees on the left above, are sometimes called corollas.

Given a graded space $X$, define a space $t(X)$ for each tree $t$ by declaring $e(X)=*$, and, if $t=\left[n ; t_{1}, \ldots, t_{n}\right]$, setting

$$
t(X)=X_{n} \times \prod_{i} t_{i}(X) .
$$

This is called the space of labelings of the tree $t$ with labels from $X$. We will denote such a labeling $l$ by $\left[x ; l_{1}, \ldots, l_{n}\right]$, where $x \in X_{n}$ and $l_{i}$ is a labeling of $t_{i}$, ie, $l_{i} \in t_{i}(X)$. We treat $t(X)$ as a graded space concentrated at level $\lambda(t)$, and let $\mathbb{T}(X)$ denote the union of the graded spaces $t(X)$, taken over all trees $t$. This is the space of trees with labels from $X$. In the pictorial terms of (4.7), a labeling of a tree by $X$ is a choice, for 
each solid dot in the sketch of the tree, of a simplex in $X_{n}$, where $n$ is the number of edges pointing inward towards the dot.

4.8 Remark For $x \in X_{n}$, it is convenient to use $[x]$ to denote the labeling $\left[x ; l_{1}, \ldots, l_{n}\right]$ in which each $l_{i}$ is the unique labeling of the trivial tree. The map $x \mapsto[x]$ gives a map $X \rightarrow \mathbb{T}(X)$ of graded spaces. Each labeling $l$ in $\mathbb{T}(X)$ has an underlying tree $\operatorname{sh}(l)$, called the shape of $l$; the labeling inherits its depth $\chi(l)$, leaf count $\lambda(l)$ and branching count $\beta(l)$ from the shape. The assignment $l \mapsto \operatorname{sh}(l)$ gives a map from $\mathbb{T}(X)$ to the graded discrete space $\mathbb{T}$ of all trees. The set Lab $(l)$ of labels in $l$ is defined by initializing $\operatorname{Lab}(*)=\varnothing$ and constructing $\operatorname{Lab}\left(\left[x ; l_{1}, \ldots, l_{n}\right]\right)$ by adjoining $x$ to the union of the sets $\operatorname{Lab}\left(l_{i}\right), 1 \leq i \leq n$. This object is a set of simplices in $X$. The assignment $l \mapsto \mathrm{Lab}(l)$ gives a map from $\mathbb{T}(X)$ to the powerset of the union $\bigcup_{n} X_{n}$.

The following proposition is elementary.

4.9 Proposition If $X$ is a graded space, $\mathbb{T}(X)$ is naturally isomorphic to the free operad $\Phi(X)$.

4.10 Remark The operad composition in $\mathbb{T}(X)$ is defined as follows. Given a labeling $a \in \mathbb{T}(X)$ with $\lambda(a)=m$ and $m$ labelings $b_{i} \in \mathbb{T}(X), 1 \leq i \leq m$, it is sufficient to specify the composite labeling $a\left(b_{1}, \ldots, b_{m}\right)$. This is given inductively by setting $*\left(b_{1}\right)=b_{1}$ and specifying that if $a=\left[x ; l_{1}, \ldots, l_{n}\right]$ then

$$
a\left(b_{1}, \ldots, b_{m}\right)=\left[x ; l_{1}\left(b_{1}, \ldots, b_{\lambda\left(l_{1}\right)}\right), \ldots, l_{n}\left(b_{m-\lambda\left(l_{n}\right)+1}, \ldots, b_{m}\right)\right] .
$$

The unique labeling of the trivial tree $e$ serves as $1_{\mathbb{T}(X)}$.

4.11 Reflexive coequalizers A reflexive pair in some category is a diagram which looks like the one-truncation of a simplicial object; more explicitly, it consists of two objects $X_{1}, X_{0}$, maps $d_{0}, d_{1}: X_{1} \rightarrow X_{0}$, and a map $s_{0}: X_{0} \rightarrow X_{1}$ such that both composites $d_{0} s_{0}$ and $d_{1} s_{0}$ give the identity map of $X_{0}$. An augmentation for the pair is an object $X$ and a map $d: X_{0} \rightarrow X$ such that $d d_{0}=d d_{1}$. The colimit of a reflexive pair is isomorphic to the coequalizer of $\left(d_{0}, d_{1}\right)$. An augmented reflexive pair is exact if the natural map from the coequalizer of $\left(d_{0}, d_{1}\right)$ to $X$ is an isomorphism, in which case the diagram is called a reflexive coequalizer diagram. Reflexive coequalizers commute with finite products in the category of sets [22, 3.2], and it follows easily from this that reflexive coequalizers of just about any algebraic species can be computed in the appropriate underlying category. See [22, 4.3] or the monograph [1]. In particular, reflexive coequalizers of operads of spaces can be computed in the category of graded spaces. 
4.12 A formula for the coproduct Let $U: \mathbf{O} \rightarrow \mathbf{S}$ be the forgetful functor, ie, the right adjoint to $\Phi$, and let $F: \mathbf{O} \rightarrow \mathbf{O}$ be the composite $\Phi U$. For any operad $\mathcal{P}$, adjointness considerations give maps $\epsilon_{\mathcal{P}}: F(\mathcal{P}) \rightarrow \mathcal{P}$ and $\sigma_{\mathcal{P}}: F(\mathcal{P}) \rightarrow F^{2}(\mathcal{P})$, and it is not hard to check that these give rise to a natural exact augmented reflexive pair $F^{2}(\mathcal{P}) \Rightarrow F(\mathcal{P}) \rightarrow \mathcal{P}$ with $d=\epsilon_{\mathcal{P}}, d_{0}=\epsilon_{F \mathcal{P}}, d_{1}=F\left(\epsilon_{\mathcal{P}}\right)$, and $s_{0}=\sigma_{\mathcal{P}}$. Let $\cup$ denote coproduct on the category of graded spaces. Since colimits commute with one another and $\Phi$ as a left adjoint commutes with colimits, it follows that for any two operads $\mathcal{P}, \mathcal{Q}$ there is a natural right exact augmented reflexive pair

$$
\Phi(F \mathcal{P} \cup F \mathcal{Q}) \Rightarrow \Phi(\mathcal{P} \cup \mathcal{Q}) \rightarrow \mathcal{P} \sqcup \mathcal{Q}
$$

We have suppressed forgetful functors here (eg, $\mathcal{P} \cup \mathcal{Q}$ really signifies $U \mathcal{P} \cup U \mathcal{Q}$ ) and in order to avoid clutter we will continue to do this in what follows. For the rest of this section we fix $\mathcal{P}$ and $\mathcal{Q}$, set $X_{1}=\Phi(F \mathcal{P} \cup F \mathcal{Q}), X_{0}=\Phi(\mathcal{P} \cup \mathcal{Q})$, and let $d_{0}, d_{1}$ and $s_{0}$ refer to the maps in the above reflexive pair involving $X_{1}$ and $X_{0}$. Note again that the operad coproduct $\mathcal{P} \sqcup \mathcal{Q}$ is isomorphic as a graded space to the coequalizer of $\left(d_{0}, d_{1}\right)$ in the category of graded spaces, because this coequalizer is reflexive.

The simplices of $X_{0}$ are labeled trees $l=\left[a ; l_{1}, \ldots, l_{n}\right]$ in which the labels, eg, $a$, range over simplices of $\mathcal{P}$ and of $\mathcal{Q}$; simplices of $X_{1}$ are labeled trees $v=\left[u ; v_{1}, \ldots, v_{n}\right]$ in which the labels are taken from $F(\mathcal{P})$ and $F(\mathcal{Q})$. The maps $d_{0}, d_{1}$ and $s_{0}$ preserve identities; their inductive descriptions are

$$
\begin{aligned}
d_{0}\left[u ; v_{1}, \ldots, v_{n}\right] & =u\left(d_{0} v_{1}, \ldots, d_{0} v_{n}\right), \\
d_{1}\left[u ; v_{1}, \ldots, v_{n}\right] & =\left[\epsilon(u) ; d_{1}\left(v_{1}\right), \ldots, d_{1}\left(v_{n}\right)\right], \\
s_{0}\left[a ; l_{1}, \ldots, l_{n}\right] & =\left[[a] ; s_{0}\left(l_{1}\right), \ldots, s_{0}\left(l_{n}\right)\right] .
\end{aligned}
$$

In each case the label $u$ is a simplex of $F \mathcal{P} \cup F \mathcal{Q}$ in level $n$, treated in the obvious way as a simplex of $\Phi(\mathcal{P} \cup \mathcal{Q})$; $a$ is a simplex of $\mathcal{P} \cup \mathcal{Q}$. In the upper formula the composition operation takes place in $\Phi(\mathcal{P} \cup \mathcal{Q})$. In the middle formula the composition operations implicitly involved in computing $\epsilon(u)$ take place either in $\mathcal{P}$ or in $\mathcal{Q}$ (depending on whether $u \in F \mathcal{P}$ or $u \in F \mathcal{Q}$ ). It is clear from the lower formula that a simplex $v$ in $X_{1}$ is in the image of $s_{0}$ if and only if each label in $v$ has branching count 1 .

4.15 An inductive construction of the coproduct It is helpful to introduce some terminology.

4.16 Definition Suppose that $\mathcal{R}$ and $\mathcal{S}$ are operads, and that $l=\left[a ; l_{1}, \ldots, l_{n}\right]$ is a nonidentity simplex of $\Phi(\mathcal{R} \cup \mathcal{S})$.

(1) $l$ has color $\mathcal{R}($ resp. color $\mathcal{S})$ if $a \in \mathcal{R}$ (resp. $a \in \mathcal{S})$, 
(2) $l$ displays adjacent colors if any $l_{i}$ does, or if any $l_{i} \neq e$ has the same color as $l$ ( $e$ itself does not display adjacent colors),

(3) $l$ is unital if $1_{\mathcal{R}} \in \operatorname{Lab}(l)$ or $1_{\mathcal{S}} \in \operatorname{Lab}(l)$, and

(4) $l$ is collapsible if $l$ displays adjacent colors or $l$ is unital.

Let $\mathbb{T}_{k}(\mathcal{P}, \mathcal{Q}) \subset \mathbb{T}(\mathcal{P} \cup \mathcal{Q})=X_{0}$ denote the graded space of all labeled trees of branching count $k$, and $\mathbb{T}_{k}^{\mathrm{c}}(\mathcal{P}, \mathcal{Q})$ the subobject of collapsible labeled trees. Let Filt $k$ be the subspace of $\mathcal{P} \sqcup \mathcal{Q}$ given by the union of the images of $\mathbb{T}_{j}(\mathcal{P}, \mathcal{Q})$ for $j \leq k$. The main proposition we use to prove homotopy invariance of coproducts is the following.

4.17 Proposition For each $k \geq 1$ there is a pushout diagram of graded spaces

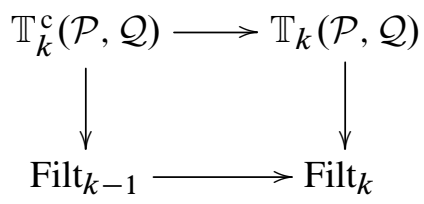

which is also a homotopy pushout diagram.

Proof of Proposition 4.3 (given Proposition 4.17) The graded space $\mathbb{T}_{k}(\mathcal{P}, \mathcal{Q})$ is homotopy invariant as a functor of $\mathcal{P}$ and $\mathcal{Q}$, since it is formed by taking disjoint unions of products of the graded constituents of these two operads. The subobject $\mathbb{T}_{k}^{\mathrm{c}}(\mathcal{P}, \mathcal{Q})$ is similarly homotopy invariant, since it is determined by selecting appropriate components (picking labeled trees that display adjacent colors) or by imposing basepoint restrictions on appropriate cartesian factors (picking unital labeled trees). Since Filt ${ }_{0}=\left\{1_{\mathcal{P} \sqcup \mathcal{Q}}\right\}$ is a single point, it follows by induction that each object Filt $k$ depends in a homotopy invariant way on $\mathcal{P}$ and $\mathcal{Q}$, and so $\mathcal{P} \sqcup \mathcal{Q}=\operatorname{colim}_{k}$ Filt $_{k}$ also does. The statement that $\mathbf{O}$ is left proper follows as in $[22,9.1]$ from the fact that the coproduct construction is homotopy invariant.

In order to obtain Proposition 4.17 from the coequalizer presentation (4.13), we will have to trim $X_{1}$ a bit, without changing the value of the graded space coequalizer (4.13). Note that the terminology of Definition 4.16 applies to $X_{1}=\mathbb{T}(F \mathcal{P} \cup F \mathcal{Q})$; moreover, the labels with which the trees in $X_{1}$ are decorated lie in $F \mathcal{P}$ or $F \mathcal{Q}$, and thus have branching counts in their own right. In the context of Definition 4.16, say that a labeled tree is alternating if it does not display adjacent colors. Define two subobjects $X_{1}^{+}$ and $X_{1}^{-}$of $X_{1}$ as follows:

- $v \in X_{1}^{+}$if $v$ is alternating, $1_{F \mathcal{P}}$ and $1_{F \mathcal{Q}}$ do not appear as labels in $v$, and at least one label in $v$ has branching count $>1$.

- $v \in X_{1}^{-}$if $v$ is alternating, $1_{F \mathcal{P}}$ or $1_{F \mathcal{Q}}$ appear among the labels, $\left[1_{\mathcal{P}}\right]$ or $\left[1_{\mathcal{Q}}\right]$ do not appear as labels, and all of the labels in $v$ have branching count $\leq 1$. 
Every $v \in X_{1}^{+}$is an alternating composite of elements of the form $[l], l \in F \mathcal{P} \cup F \mathcal{Q}$, such that no $l$ is $1_{F \mathcal{P}}$ or $1_{F \mathcal{Q}}$ and at least one $l$ is itself a nontrivial composite (in $F \mathcal{P}$ or $F \mathcal{Q}$ ). Every element in $X_{1}^{-}$is an alternating composite of the elements $\left[1_{F \mathcal{P}}\right]$ and $\left[1_{F \mathcal{Q}}\right]$ (at least one of which must appear) with elements of the form $[[a]]$ where $a \in \mathcal{P} \cup \mathcal{Q}$ and $a$ does not equal $1_{\mathcal{P}}$ or $1_{\mathcal{Q}}$.

It may be helpful in understanding these definitions is to think of the case in which $\mathcal{P}$ and $\mathcal{Q}$ are graded discrete objects concentrated at level 1, equivalently, classic monoids (Section 7). The free monoid on a set $A$ has as elements the sequences $\left(a_{1}, \ldots, a_{k}\right)$ $(k \geq 0)$ in which the terms $a_{i}$ are taken from $A$ (in our language, this is a labeling of a linear tree: the $a_{i}$ 's are the labels, and the sequence length $k$ is the branching count). Multiplication is given by concatenation; the empty sequence is allowed, and serves as the identity element. Then $X_{0}$ has as elements the sequences above in which each $a_{i}$ is taken either from $\mathcal{P}$ or from $\mathcal{Q}$. Similarly $X_{1}$ is made up of sequences $v=\left(u_{1}, \ldots, u_{k}\right),(k \geq 0)$, such that each $u_{i}$ itself is either a sequence of elements from $\mathcal{P}$ or a sequence of elements from $\mathcal{Q}$. Note that the empty sequence of elements in $\mathcal{P}\left(1_{F \mathcal{P}}\right)$ is to be distinguished from the empty sequence of elements in $\mathcal{Q}\left(1_{F \mathcal{Q}}\right)$. The map $d_{0}$ concatenates the sequences $u_{i}$ together; this usually results in a longer sequence, but not always, because both varieties $1_{F \mathcal{P}}$ and $1_{F \mathcal{Q}}$ of empty sequence collapse when the concatenation is performed. The map $d_{1}$ contracts each sequence $u_{i}$ by multiplying it out (either in $\mathcal{P}$ or $\mathcal{Q}$, as appropriate) to a single element $\bar{u}_{i}$, thus obtaining a sequence $\left(\bar{u}_{1}, \ldots, \bar{u}_{k}\right)$ in $X_{0}$. A sequence $v$ belongs to $X_{1}^{+}$if the $u_{i}$ alternate in being sequences from $\mathcal{P}$ and sequences from $\mathcal{Q}$, no $u_{i}$ is an empty sequence, and at least one $u_{i}$ is a sequence of length $>1$. This guarantees that the length of $d_{0}(u)$ is greater than the length of $u$, while the length of $d_{1}(u)$ equals the length of $u$. A sequence $u$ belongs to $X_{1}^{-}$if the $u_{i}$ again alternate, at least one $u_{i}$ is an empty sequence, each of the $u_{i}$ has length $\leq 1$, and no $u_{i}$ is a length 1 sequence consisting solely of $1_{\mathcal{P}}$ or $1_{\mathcal{Q}}$. This guarantees among other things that the length of $d_{0} v$ is less than the length of $v$, while the length of $d_{1}(v)$ equals the length of $v$. These length (branching count) considerations play a role in the proof of Proposition 4.17.

Given a graded subspace $Y \subset X_{1}$, and $l, l^{\prime} \in X_{0}$, write $l \sim_{Y} l^{\prime}$ and say that $l$ is congruent to $l^{\prime} \bmod Y$ if $l$ and $l^{\prime}$ have the same image in the $\mathbf{S}$-coequalizer of $\left(\left.d_{0}\right|_{Y},\left.d_{1}\right|_{Y}\right)$. Say that $Y$ is saturated if the equivalence relation $\sim_{Y}$ respects operad composition in $X_{0}$. Let $X_{1}^{ \pm}=X_{1}^{+} \cup X_{1}^{-}$.

4.18 Lemma The graded subspace $X_{1}^{ \pm} \subset X_{1}$ is saturated.

Proof This is a straightforward calculation, but writing it out in detail is tedious. Each label in $X_{0}$ is either from $\mathcal{P}$ or from $\mathcal{Q}$. Congruence mod $X_{1}^{+}$allows adjacent labels 
of the same color to be composed, so that every simplex of $X_{0}$ is congruent $\bmod X_{1}^{+}$to a unique alternating element. Now observe that for $v=\left[1_{F \mathcal{P}}\right]$, for instance, $d_{0} v=1_{X_{0}}$ while $d_{1} v=\left[1_{\mathcal{P}}\right]$. This implies that additionally allowing congruence $\bmod X_{1}^{-}$permits leaving out terms of the form $\left[1_{\mathcal{P}}\right]$ or $\left[1_{\mathcal{Q}}\right]$ from an alternating element of $X_{0}$. Of course, excising such a term renders the element nonalternating, and so it must be reduced further by congruence mod $X_{1}^{+}$. (This reduction might introduce other terms of the form $\left[1_{\mathcal{P}}\right]$ or $\left[1_{\mathcal{Q}}\right]$ since some composite of elements in $\mathcal{P}_{1}$ for instance, might be $1_{\mathcal{P}}$, but the reduction process is bound to stop eventually.) The upshot is that every labeled tree in $X_{0}$ is congruent $\bmod X_{1}^{ \pm}$to a unique alternating labeled tree which contains no labels of the form $1_{\mathcal{P}}$ or $1_{\mathcal{Q}}$. Compatibility with composition is not hard to check inductively.

Let $d_{0}^{ \pm}$and $d_{1}^{ \pm}$denote the restrictions of $d_{0}$ and $d_{1}$ to $X_{1}^{ \pm}$. In the statement of the following lemma, coequalizers are to be computed in $\mathbf{S}$.

4.19 Lemma The natural map from the coequalizer of $\left(d_{0}^{ \pm}, d_{1}^{ \pm}\right)$to the coequalizer of $\left(d_{0}, d_{1}\right)$ is an isomorphism of graded spaces.

Proof Since $X_{1}^{ \pm}$is saturated, it is enough to prove that if $v \in X_{1}$ has branching count 1 , then $d_{0} v$ is congruent to $d_{1} v \bmod X_{1}^{ \pm}$. But this is obvious: either $v=\left[1_{F \mathcal{P}}\right]$ or $\left[1_{F \mathcal{Q}}\right]$ (in which case $v \in X_{1}^{-}$), $v=[[a]]$ for $a \in \mathcal{P} \cup \mathcal{Q}$ (in which case $d_{0} v=d_{1} v$ ), or $v=[l]$ for some $l \in F \mathcal{P} \cup F \mathcal{Q}$ of complexity $>1$, in which case $v \in X_{1}^{+}$.

Proof of Proposition 4.17 Let $f$ (resp. $g$ ) be the map $X_{1}^{ \pm} \rightarrow X_{0}$ given by $d_{0}$ on $X_{1}^{+}$and $d_{1}$ on $X_{1}^{-}$(resp. $d_{1}$ on $X_{1}^{+}$and $d_{0}$ on $X_{1}^{-}$). By Lemma 4.19, $\mathcal{P} \sqcup \mathcal{Q}$ is the coequalizer of $(f, g)$. It is easy to check that $f$ raises branching count on $X_{1}^{+}$ and preserves branching count on $X_{1}^{-}$, whereas $g$ preserves this invariant on $X_{1}^{+}$ and lowers it on $X_{1}^{-}$. The effect of the coequalizer is thus to identify trees of high branching count in the image of $f$ with trees of lower branching count in the image of $g$. Observe that a simplex of $\mathbb{T}_{k}(\mathcal{P}, \mathcal{Q})$ is in $f\left(X_{1}^{ \pm}\right)$if and only if it belongs to $\mathbb{T}_{k}^{c}(\mathcal{P}, \mathcal{Q})$. The first statement of the proposition now follows from relatively routine considerations. The fact that the pushout is a homotopy pushout is a consequence of the fact that the upper arrow is a cofibration.

Proof of Proposition 4.4 The fact that $\Phi$ preserves equivalences follows from Proposition 4.9. To see that $\Phi^{e}$ also preserves equivalences, note that if $X$ is a pointed graded space, there is an exact augmented split coequalizer diagram $X_{++} \Rightarrow X_{+} \rightarrow X$ in the category of pointed graded spaces, obtained by combining the forgetful functor from pointed objects to unpointed ones with its left adjoint $(-)_{+}$. Applying $\Phi^{e}$, using 
the fact that (as a left adjoint) $\Phi^{e}$ commutes with colimits, and observing that for, any $Y, \Phi^{e}\left(Y_{+}\right)=\Phi(Y)$, gives an augmented reflexive coequalizer diagram

$$
\Phi\left(X_{+}\right) \Rightarrow \Phi(X) \rightarrow \Phi^{e}(X)
$$

which is exact both in $\mathbf{O}$ and in $\mathbf{S}$. One of the arrows $\Phi\left(X_{+}\right) \rightarrow \Phi(X)$ in this diagram preserves branching count, the other lowers it. We leave it to the reader to exploit this in order to give an inductive homotopy invariant construction for $\Phi^{e}(X)$ along the lines of Proposition 4.17.

\section{Resolutions of bimodules and operads}

In this section we describe a particular operad resolution that will play a key role in Section 6. This resolution begins as a resolution of a bimodule Proposition 5.2, but it is promoted to a resolution of an operad by application of an enveloping functor Proposition 5.4.

First, the enveloping functor. From now on in this section, $\mathcal{P}$ and $\mathcal{Q}$ are chosen operads. The category $\mathbf{S}_{\mathcal{P}, \mathcal{Q}}^{e}$ is the category of pointed $\mathcal{P}-\mathcal{Q}$-bimodules; an object $X$ of this category has a basepoint in level 1, or equivalently, is supplied with a bimodule map $\mathcal{P} \circ \mathcal{Q} \rightarrow X$. Similarly, $\mathbf{O}_{\mathcal{P}, \mathcal{Q}}$ is the category $(\mathcal{P} \sqcup \mathcal{Q}) \searrow \mathbf{O}$ of operads under $\mathcal{P}$ and $\mathcal{Q}$. Retaining the identity element as basepoint gives a forgetful functor $\downarrow: \mathbf{O}_{\mathcal{P}, \mathcal{Q}} \rightarrow \mathbf{S}_{\mathcal{P}, \mathcal{Q}}^{e}$. The following proposition is elementary (it's easy to describe values of the left adjoint by generators and relations).

\subsection{Proposition The forgetful functor $\llbracket: \mathbf{O}_{\mathcal{P}, \mathcal{Q}} \rightarrow \mathbf{S}_{\mathcal{P}, \mathcal{Q}}^{e}$ has a left adjoint $E$.}

From now on in this section, $\mathcal{P}$ is a fixed operad. The Hochschild resolution of $\mathcal{P}$ as a bimodule over itself is the simplicial pointed bimodule $\mathrm{H}(\mathcal{P})$ with

$$
\mathrm{H}_{n}(\mathcal{P})=\mathcal{P}^{\circ(n+2)} .
$$

The object on the right is a composition power of $\mathcal{P}$, with the evident left and right actions. The face map $d_{i}$ is given by using operad multiplication to combine factors $i+1$ and $i+2$ in $\mathcal{P}^{\circ(n+2)}$, and the degeneracy map $s_{i}$ is given by using the unit inclusion $e_{\circ} \rightarrow \mathcal{P}$ to insert the unit between factor $i+1$ and factor $i+2$. The images of $\mathrm{H}_{0}(\mathcal{P})$ under the degeneracy maps provide the necessary basepoints.

The diagonal $\mathrm{dH}(\mathcal{P})$ is the $\mathcal{P}-\mathcal{P}$-bimodule whose $n$-simplices are the $n$-simplices of $\mathrm{H}_{n}(\mathcal{P})$. Operad multiplication maps $\mathcal{P}^{\circ n} \rightarrow \mathcal{P}$ induce a bimodule map $\alpha: \mathrm{dH}(\mathcal{P}) \rightarrow \mathcal{P}$, and the basepoints $\mathcal{P} \circ \mathcal{P} \rightarrow \mathrm{H}_{n}(\mathcal{P})$ pass to a basepoint $\mathcal{P} \circ \mathcal{P} \rightarrow \mathrm{dH}(\mathcal{P})$. 
5.2 Proposition The bimodule $\mathrm{dH}(\mathcal{P})$ is a cofibrant object of $\mathbf{S}_{\mathcal{P}, \mathcal{P}}^{e}$, and the map $\mathrm{dH}(\mathcal{P}) \rightarrow \mathcal{P}$ above is an equivalence.

Proof The first statement amounts to a claim that $\mathcal{P} \circ \mathcal{P} \rightarrow \mathrm{dH}(\mathcal{P})$ is a cofibration of $\mathcal{P}-\mathcal{P}$-bimodules, and this is immediate from the description of cofibrations in Remark 4.2. The second one is as usual a consequence of the fact that, after forgetting from simplicial bimodules to simplicial graded spaces, there are extra degeneracy maps $s_{-1}: \mathrm{H}_{n}(\mathcal{P}) \rightarrow \mathrm{H}_{n+1}(\mathcal{P})$ which insert the unit as the first composition factor.

Applying the functor $E$ of Proposition 5.1 to $\mathrm{H}(\mathcal{P})$ degree by degree gives a simplicial operad under $\mathcal{P} \sqcup \mathcal{P}$. The diagonal $d E \mathrm{H}(\mathcal{P})$ is then an operad under $\mathcal{P} \sqcup \mathcal{P}$, and too is also supplied with a map $d E \mathrm{H}(\mathcal{P}) \rightarrow \mathcal{P}$ which factors the fold map $\mathcal{P} \sqcup \mathcal{P} \rightarrow \mathcal{P}$. This last is a consequence of the easily verified fact that if $\mathcal{P}$ is treated as a pointed bimodule over itself in the natural way, $E(\mathcal{P})$ is isomorphic as an operad under $\mathcal{P} \sqcup \mathcal{P}$ to $\mathcal{P}$ itself, supplied with the fold map $\mathcal{P} \sqcup \mathcal{P} \rightarrow \mathcal{P}$. We now observe that the same result can be obtained by applying $E$ to the diagonal bimodule $\mathrm{dH}(\mathcal{P})$.

5.3 Proposition There two operads $E(\mathrm{dH}(\mathcal{P}))$ and $d E \mathrm{H}(\mathcal{P})$ are isomorphic in a natural way (in the category of operads under $\mathcal{P} \sqcup \mathcal{P}$ and over $\mathcal{P}$ ).

Proof Let $\mathcal{P}_{k}$ be the operad of sets obtained by taking the dimension $k$ simplices of $\mathcal{P}$, and $E_{k}$ the left adjoint to the forgetful functor from operads under $\mathcal{P}_{k} \sqcup \mathcal{P}_{k}$ to $\mathcal{P}_{k}-\mathcal{P}_{k}$-bimodules. It is elementary to see that the functor $E$ is cobbled together from the functors $E_{k}$; in other words, if $X$ is a pointed bimodule over $\mathcal{P}$ and $X_{k}$ is the bimodule over $\mathcal{P}_{k}$ obtained by taking the collection of $k$-simplices in $X$, the $E(X)$ is an operad in simplicial sets with $E(X)_{k}=E_{k}\left(X_{k}\right)$. The result follows immediately.

5.4 Proposition The operad $d E \mathrm{H}(\mathcal{P})$ is a cofibrant object of $\mathbf{O}_{\mathcal{P}, \mathcal{P}}$, and the map $d E \mathrm{H}(\mathcal{P}) \rightarrow \mathcal{P}$ is an equivalence.

5.5 Remark The diagonal principle which figures in the following proof states that if $X \rightarrow Y$ is a map of simplicial (graded) spaces which is an equivalence in each simplicial degree, then the induced map $\operatorname{diag} X \rightarrow \operatorname{diag} Y$ is an equivalence [8, IV.1.7].

If $S$ is a simplicial set and $A$ is an object in some category with coproducts, we let $S \cdot A$ denote the coproduct of $S$ copies of $A$. This construction is functorial in $S$, and so for a simplicial set $K$ there is a simplicial object $K \cdot A$ which in simplicial degree $n$ consists of $K_{n} \cdot A$. If $A$ is itself a simplicial object, then $K \cdot A$ denotes the diagonal of the bisimplicial object $\left\{K_{i} \cdot A_{j}\right\}$. 
Proof of Proposition 5.4 The functor $E$ is left adjoint to a functor which preserves fibrations and equivalences, and so $E$ preserves cofibrant objects and equivalences between cofibrant objects 2.9. The statement that $d E \mathrm{H}(\mathcal{P})$ is cofibrant now follows from Propositions 5.3 and 5.2.

Say that the operad $\mathcal{P}$ is $\operatorname{good}$ if $d E \mathrm{H}(\mathcal{P}) \rightarrow \mathcal{P}$ is an equivalence. There is an isomorphism

$$
E \mathrm{H}_{n}(\mathcal{P}) \cong E \mathcal{P}^{\circ(n+2)} \cong \mathcal{P} \sqcup \Phi^{e}\left(\mathcal{P}^{\circ n}\right) \sqcup \mathcal{P},
$$

and so it follows from Propositions 4.3 and 4.4 that $E \mathrm{H}_{n}(\mathcal{P})$ depends on $\mathcal{P}$ in a homotopy invariant way. Hence (by the diagonal principle) if $\mathcal{P} \rightarrow \mathcal{Q}$ is an equivalence, then $\mathcal{Q}$ is good if and only if $\mathcal{P}$ is. In particular, in order to check whether $\mathcal{P}$ is good, we may assume that $\mathcal{P}$ is cofibrant as an operad (Remark 4.2). Another application of the diagonal principle (cf the proof of Proposition 5.3) shows that $\mathcal{P}$ is good if for each $n \geq 0$ the discrete operad $\mathcal{P}_{n}$ is good, where $\mathcal{P}_{n}$ is the operad of $n$-simplices in $\mathcal{P}$. The upshot is that in order to prove that any operad $\mathcal{P}$ is good, it is enough to treat the special case in which $\mathcal{P}$ is the free operad $\Phi X$ on a graded set (discrete graded space) $X$.

Given $\mathcal{P}=\Phi X$, we identify $\mathrm{H}(\mathcal{P})$ with $\mathrm{dH}(\mathcal{P})$, since $\mathrm{H}(\mathcal{P})$ is constant in the internal simplicial direction. We will construct a cofibrant object $J$ of $\mathbf{S}_{\mathcal{P}, \mathcal{P}}^{e}$ together with an equivalence $J \rightarrow \mathrm{H}(\mathcal{P})$, such that $E(J)$ is clearly equivalent to $\mathcal{P}$. The proposition will follow from the fact that $E$ preserves equivalences between cofibrant bimodules (Remark 3.3).

There is a natural map $X \rightarrow \mathcal{P}$ of graded spaces (the inclusion of generators), and so a forgetful functor $\Psi$ from pointed $\mathcal{P}-\mathcal{P}$-bimodules to the category of graded spaces under $X \cup X$. Let $G$ be left adjoint to $\Psi$. Applying $(-) \cdot X$ to the inclusion $\partial \Delta[1] \rightarrow \Delta[1]$ gives $\Delta[1] \cdot X$ the structure of a graded space under $X \cup X$. Since $\Delta[1] \cdot X$ is free as a simplicial graded set on a single copy of the graded set $X$ in dimension 1 , there is a map $\Delta[1] \cdot X \rightarrow \Psi \mathrm{H}(\mathcal{P})$ determined by

$$
X \rightarrow \mathrm{H}_{1}(\mathcal{P}) \cong \mathcal{P} \circ \mathcal{P} \circ \mathcal{P}, \quad x \mapsto e([x](e, \ldots, e)),
$$

where the number of copies of $e$ on the right equals the level of $x$. The object $J$ is $G(\Delta[1] \cdot X)$; adjointness gives a map $J \rightarrow \mathrm{H}(\mathcal{P})$. We claim that this map is an equivalence, that $J$ is cofibrant, and that $E(J) \sim \mathcal{P}$.

By inspection, in dimension $n$ the object $J$ is isomorphic to $\mathcal{P} \circ\left(X^{\cup n}\right)_{+} \circ \mathcal{P}$, where $(-)_{+}$signifies adding a disjoint basepoint at level 1 ; from this it easily follows that $J$ is cofibrant as a pointed bimodule of Remark 4.2. Again by inspection, $E(J)$ is isomorphic to $\Delta[1] \cdot \mathcal{P}$ (calculated in $\mathbf{O}$ ) or equivalently to $\Phi(\Delta[1] \cdot X)$; since $\Delta[1]$ is 
simplicially contractible to either boundary vertex, $E(J)$ is simplicially contractible to $\mathcal{P}$.

It remains to show that $J \rightarrow \mathrm{H}(\mathcal{P})$ is an equivalence. To do so we prove that the map $J \rightarrow \mathcal{P}$, given by the multiplication map in degree 0 , is an equivalence. Since this map clearly factors through the equivalence $\mathrm{H}(\mathcal{P}) \rightarrow \mathcal{P}$, we can then conclude that $J \rightarrow \mathrm{H}(\mathcal{P})$ is an equivalence as well. It is easy to check that $\pi_{0} J$, which is the coequalizer of the two maps $d_{0}, d_{1}: \mathcal{P} \circ X_{+} \circ \mathcal{P} \rightarrow \mathcal{P} \circ \mathcal{P}$ is in fact isomorphic to $\mathcal{P}$ via the operad multiplication $\mathcal{P} \circ \mathcal{P} \rightarrow \mathcal{P}$, so it is enough to check that for each labeled tree $l \in \mathcal{P}=\mathbb{T}(X)$, the component $C(l)$ of $J$ corresponding to $l$ is contractible. We show this by induction on the branching count of $l$. The complex $C(e)$ is isomorphic to $\Delta[0]$. Suppose that $l=\left[x ; l_{1}, \ldots, l_{n}\right]$. The $k$-simplices of $C(l)$ prescribe ways of splitting $l$ as a three-fold composition

$$
l=p_{0}\left(\left[x_{1}\right]\left(p_{1}^{1}, \ldots, p_{j_{1}}^{1}\right), \ldots,\left[x_{m}\right]\left(p_{1}^{m}, \ldots, p_{j_{m}}^{m}\right)\right),
$$

where $p_{0}$ and the $p_{a}^{b}$ 's are elements of $\mathcal{P}, m$ is the level of $p_{0}$, the $x_{i}$ 's belong to $\left(X^{\cup k}\right)_{+}$, and $j_{i}$ is the level of $x_{i}$. (The rogue element $[+]$, where + is the disjoint basepoint, is to be treated in the composition formula as the identity element $e=1_{\mathcal{P}}$.) Consider the following two possibilities. If $p_{0}$ is not $e$, then this simplex lies in the image of the monomorphism

$$
C\left(l_{1}\right) \times \cdots \times C\left(l_{n}\right) \rightarrow C(l), \quad\left(\sigma_{1}, \ldots, \sigma_{n}\right) \mapsto[x]\left(\sigma_{1}, \ldots, \sigma_{n}\right),
$$

where $[x]\left(\sigma_{1}, \ldots, \sigma_{n}\right)$ involves the left action of $\mathcal{P}$ on $J$. If $p_{0}=e$, then the simplex lies in the image of

$$
\Delta[1] \rightarrow C(l), \quad \Delta_{1} \mapsto e \circ x \circ\left(l_{1}, \ldots, l_{n}\right),
$$

where $\Delta_{1}$ is the generating one-simplex of $\Delta[1]$. These two images are contractible (the first by induction), they cover $C(l)$, and they overlap in the zero-simplex $[x] \circ[+] \circ\left(l_{1}, \ldots, l_{n}\right)$. It follows that $C(l)$ is contractible.

\section{Distinguished objects and the proof of Theorem 1.9}

In this section we prove a key technical result Proposition 6.1 which leads at the end of the section to a proof of Theorem 1.9. We continue to use the notation of Section 5, with the convention that $\mathcal{P}$ and $\mathcal{Q}$ are fixed operads. An object $X$ of $\mathbf{S}_{\mathcal{P}, \mathcal{Q}}^{e}$ is distinguished if the natural map $\mathcal{Q} \rightarrow X$ is an equivalence, and an object $\mathcal{R}$ of $\mathbf{O}_{\mathcal{P}, \mathcal{Q}}$ is distinguished if $\mathcal{Q} \rightarrow \mathcal{R}$ is an equivalence. Note that both $\mathbf{S}_{\mathcal{P}, \mathcal{Q}}^{e}$ and $\mathbf{O}_{\mathcal{P}, \mathcal{Q}}$ inherit model structures from Proposition 4.1. The functor $E^{\mathrm{h}}: \mathbf{S}_{\mathcal{P}, \mathcal{Q}}^{e} \rightarrow \mathbf{O}_{\mathcal{P}, \mathcal{Q}}$ is the left derived functor from Remark 3.3 of $E$ (see Proposition 5.1). 


\subsection{Proposition The functor $E^{\mathrm{h}}$ preserves distinguished objects.}

We will build up to this in stages. It is convenient to denote a pointed $\mathcal{P}-\mathcal{Q}$-bimodule $X$ by writing out the triple $T=(\mathcal{P}, X, \mathcal{Q})$, where the basepoint $e_{\mathrm{O}} \rightarrow X$ is understood. A morphism of triples consists of three morphisms, two of operads and one of pointed bimodules, which are compatible in the obvious sense. The morphism is an equivalence if all of its constituents are equivalences. The functors $E$ and $E^{\mathrm{h}}$ extend to functors on the category of triples.

6.2 Proposition If $f: T \rightarrow T^{\prime}$ is an equivalence of triples, then $E^{\mathrm{h}}(f)$ is an equivalence of operads. In particular, if $E^{\mathrm{h}}(T)$ is distinguished, so is $E^{\mathrm{h}}\left(T^{\prime}\right)$.

Proof Write $T=(\mathcal{P}, X, \mathcal{Q})$ and $T^{\prime}=\left(\mathcal{P}^{\prime}, X^{\prime}, \mathcal{Q}^{\prime}\right)$. Let $f^{*}: \mathbf{S}_{\mathcal{P}^{\prime}, \mathcal{Q}^{\prime}}^{e} \rightarrow \mathbf{S}_{\mathcal{P}, \mathcal{Q}}^{e}$ be the restriction functor and $f_{*}$ its left adjoint. According to $[22,8.6]$, the pair $\left(f_{*}, f^{*}\right)$ is a Quillen equivalence. Similarly, let $g$ be the map $\mathcal{P} \sqcup \mathcal{Q} \rightarrow \mathcal{P}^{\prime} \sqcup \mathcal{Q}^{\prime}$, $g^{*}: \mathbf{O}_{\mathcal{P}^{\prime}, \mathcal{Q}^{\prime}} \rightarrow \mathbf{O}_{\mathcal{P}, \mathcal{Q}}$ the restriction map, and $g_{*}$ its left adjoint. The map $g$ is an equivalence (see Proposition 4.3) and so according to [22, 2.7] it follows from the fact that $\mathbf{O}$ is left proper by Proposition 4.3 that the pair $\left(g_{*}, g^{*}\right)$ is a Quillen equivalence. It is easy to see that $f_{*}\left(X^{\mathrm{c}}\right) \rightarrow X^{\prime}$ is an equivalence (cf [22, Proof of 8.6]), and so, since $f_{*}\left(X^{\mathrm{c}}\right)$ is a cofibrant object equivalent to $X^{\prime}, E^{\mathrm{h}}\left(X^{\prime}\right) \sim E f_{*}\left(X^{\mathrm{c}}\right)$. Uniqueness of adjoints implies that $E f_{*} \cong g_{*} E$, and the proposition follows directly.

A triple $(\mathcal{P}, \mathcal{Q}, \mathcal{Q})$ is obtained from a homomorphism $f: \mathcal{P} \rightarrow \mathcal{Q}$ if the basepoint in $\mathcal{Q}$ is the operad unit, the right action of $\mathcal{Q}$ on itself is the usual one, and the left action of $\mathcal{P}$ on $\mathcal{Q}$ is obtained by composing the usual left action of $\mathcal{Q}$ on itself with the morphism $f$. Such a triple is automatically distinguished.

6.3 Proposition If a triple $T$ is obtained from $f: \mathcal{P} \rightarrow \mathcal{Q}$, then $E^{\mathrm{h}}(T)$ is distinguished.

6.4 Lemma $A$ cofibration $X \rightarrow Y$ of cofibrant $\mathcal{P}-\mathcal{Q}$-bimodules is also a cofibration of cofibrant right $\mathcal{Q}$ modules. The same statement holds for pointed bimodules.

Proof According to Remark 4.2, up to retracts the hypotheses amount to the condition that there are free degeneracy objects $A \subset B \subset Y$ in the category of graded sets such that $A \subset X$, and such that the natural maps $\mathcal{P} \circ A \circ \mathcal{Q} \rightarrow X$ and $\mathcal{P} \circ B \circ \mathcal{Q} \rightarrow Y$ are isomorphisms of degeneracy objects. (Note that as in [22, Proof of 6.2(2)], it is automatic that any basis for $A$ extends to a basis for $B$.) In the pointed case, the degeneracies of the basepoint are required to lie in $A$. It is enough to show that $\mathcal{P} \circ A$ 
and $\mathcal{P} \circ B$ are free degeneracy objects in the category of graded sets such that some basis for $\mathcal{P} \circ A$ can be extended to a basis for $\mathcal{P} \circ B$. In light of the nature of the composition product (1.3) and of the fact that as a degeneracy diagram each simplicial set $\mathcal{P}_{n}$ is free [22, Section 6], the proof comes down to the remark that if $S \subset T$ is an inclusion of free degeneracy diagrams of sets, and $R$ is another such free degeneracy diagram, then $S \times R \rightarrow T \times R$ is also an inclusion of free degeneracy diagrams. This is proved by observing that if $D_{i}$ is a free degeneracy diagram on a element of dimension $i$ then $D_{i}$ is isomorphic to the diagram of simplices in $\Delta[i]$ which do not lie on $\partial \Delta[i]$; it follows that $D_{i} \times D_{j}$ is the free degeneracy diagram formed by the simplices of $\Delta[i] \times \Delta[j]$ which do not lie on the boundary of this product. A basis for $D_{i} \times D_{j}$ can be described in terms of shuffles $[18,6.5]$.

Proof of Proposition 6.3 Let $f^{*}: \mathbf{S}_{\mathcal{P}, \mathcal{Q}}^{e} \rightarrow \mathbf{S}_{\mathcal{P}, \mathcal{P}}^{e}$ be the restriction functor and $f_{*}$ its left adjoint. Similarly, let $g^{*}: \mathbf{O}_{\mathcal{P}, \mathcal{Q}} \rightarrow \mathbf{O}_{\mathcal{P}, \mathcal{P}}$ be the restriction functor and $g_{*}$ its left adjoint. As in the proof of Proposition 6.2, we have $E f_{*} \cong g_{*} E$. The functors $f_{*}$ and $g_{*}$ preserve cofibrations and equivalences between cofibrant objects (Section 2.9); moreover, in view of the formula $f_{*}(X) \cong X \circ_{\mathcal{P}} \mathcal{Q}[22,4.4,4.7], f_{*}$ is effectively left adjoint to the restriction functor from right $\mathcal{Q}$-modules to right $\mathcal{P}$-modules, and so preserves equivalences between objects which are cofibrant as right $\mathcal{P}$-modules.

Let $\mathcal{P}^{\mathrm{c}}$ be a cofibrant replacement for $\mathcal{P}$ as a pointed $\mathcal{P}-\mathcal{P}$-bimodule. Since $\mathcal{P}$ is cofibrant as a right $\mathcal{P}$-module, the observation above implies the map $f_{*}\left(\mathcal{P}^{\mathrm{c}}\right) \rightarrow f_{*}(\mathcal{P}) \cong \mathcal{Q}$ is an equivalence, so $E^{\mathrm{h}}(\mathcal{Q}) \sim E f_{*}\left(\mathcal{P}^{\mathrm{c}}\right) \cong g_{*} E\left(\mathcal{P}^{\mathrm{c}}\right)$. Moreover, $E\left(\mathcal{P}^{\mathrm{c}}\right)$ is distinguished (see Propositions 5.3 and 5.4). Consider the diagram

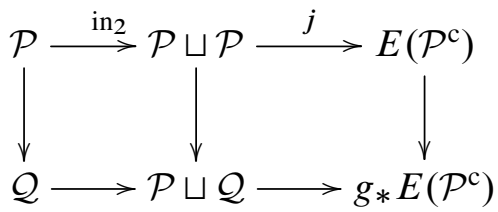

in which the upper composite is an equivalence. The small squares are cocartesian as well as homotopy cocartesian $\left[8,\left(\right.\right.$ II.8.14) ${ }^{\text {op }}$ ff]. The homotopy cocartesian property for the left-hand square is a consequence of 4.3 and for the right-hand square it is a consequence of the definition [8, (II.8.14) ${ }^{\mathrm{op}}$ ] and the fact that $j$ is a cofibration of operads ( $E$ preserves cofibrant objects). By [8, (II.8.22) $\left.{ }^{\mathrm{op}}\right]$ the large rectangle is homotopy cocartesian, and the result follows easily. 
Recall from Section 1.10 that category of right $\mathcal{Q}$-modules is closed under the graded cartesian product operation $\odot$. The endomorphism operad $\mathcal{E}_{\mathcal{Q}}(M)$ of a right $\mathcal{Q}-$ module $M$ is given by

$$
\mathcal{E}_{\mathcal{Q}}(M)_{n}=\operatorname{Map}_{\mathcal{Q}}\left(M^{\odot n}, M\right) .
$$

The subscript $n$ on the left denotes the level of the graded space forming the operad, and the object on the right is a simplicial set of right $\mathcal{Q}$-module maps. The object $\mathcal{E}_{\mathcal{Q}}(M)$ is an operad, and $M$ is naturally an $\mathcal{E}_{\mathcal{Q}}(M)-\mathcal{Q}$-bimodule.

6.5 Remark In the setting above, the $\mathcal{P}-\mathcal{Q}$-bimodule structures on $M$ which extend the given right $\mathcal{Q}$ structure are in bijective correspondence with operad homomorphisms $\mathcal{P} \rightarrow \mathcal{E}_{\mathcal{Q}}(M)$. If $\mathcal{Q}$ is treated as a right module over itself in the usual way, then the left action of $\mathcal{Q}$ on itself induces an isomorphism of operads $\mathcal{Q} \cong \mathcal{E}_{\mathcal{Q}}(\mathcal{Q})$. This follows from the fact that $\mathcal{Q}^{\odot n} \cong\left(*_{n}\right) \circ \mathcal{Q}$ is the free right $\mathcal{Q}$-module on a single point $\left(*_{n}\right)$ at level $n$.

If $f: M \rightarrow N$ is a map of right $\mathcal{Q}$-modules, the endomorphism operad $\mathcal{E}(f)$ of $f$ is constructed by letting $\mathcal{E}(f)_{n}$ be the simplicial mapping space of right $\mathcal{Q}$-module maps $f^{\odot n} \rightarrow f$; this is the space of all commutative diagrams

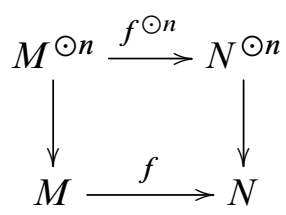

in which the vertical maps respect the right $\mathcal{Q}$-actions. There are natural operad maps $\mathcal{E}(f) \rightarrow \mathcal{E}(M)$ and $\mathcal{E}(f) \rightarrow \mathcal{E}(N)$.

6.6 Lemma If $f: \mathcal{Q} \rightarrow N$ is an acyclic cofibration of right $\mathcal{Q}$-modules such that $N$ is fibrant, then the natural operad maps $\mathcal{E}_{\mathcal{Q}}(f) \rightarrow \mathcal{E}_{\mathcal{Q}}(\mathcal{Q})$ and $\mathcal{E}_{\mathcal{Q}}(f) \rightarrow \mathcal{E}_{\mathcal{Q}}(N)$ are equivalences.

Proof For each $n \geq 0$ there is a fibre square

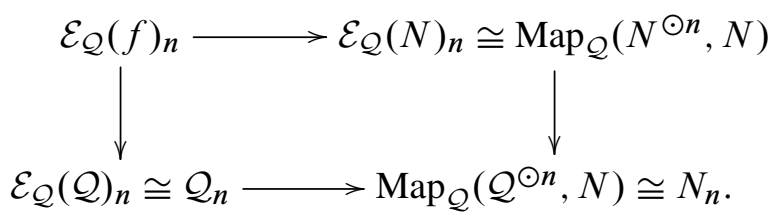

For the identifications on the bottom row, recall from Remark 6.5 that $\mathcal{Q}^{\odot n}$ is the free right $\mathcal{Q}$-module on a generator at level $n$. The lower map is an equivalence, 
and so it is enough to show that the right vertical map is an acyclic fibration, or even that $\mathcal{Q}^{\odot n} \rightarrow N^{\odot n}$ is an acyclic cofibration of right $\mathcal{Q}$-modules. This latter map is clearly an equivalence. The required cofibration statement follows from Remark 4.2, the distributive formula (1.11), and the fact that the product of two free degeneracy diagrams of sets is again a free degeneracy diagram (proof of Lemma 6.4).

Proof of Proposition 6.1 Suppose that $M$ is a distinguished $\mathcal{P}-\mathcal{Q}$-bimodule. By the homotopy invariance of $E^{\mathrm{h}}$ we can assume that $M$ is fibrant and cofibrant as a pointed bimodule, so that in particular the map $\mathcal{P} \circ \mathcal{Q} \rightarrow M$ provided by the basepoint is a cofibration. Let $f: \mathcal{Q} \rightarrow \mathcal{P} \circ \mathcal{Q} \rightarrow M$ be the right $\mathcal{Q}$-module map provided by the basepoint; $f$ is a cofibration of right $\mathcal{Q}$-modules (Lemma 6.4) and the natural operad maps $\mathcal{E}_{\mathcal{Q}}(f) \rightarrow \mathcal{E}_{\mathcal{Q}}(M)$ and $\mathcal{E}_{\mathcal{Q}}(f) \rightarrow \mathcal{Q}$ (Remark 6.5) are equivalences by Lemma 6.6. The left action of $\mathcal{P}$ on $M$ gives an operad homomorphism $\mathcal{P} \rightarrow \mathcal{E}_{\mathcal{Q}}(M)$ which we factor as the composite of an acyclic cofibration $\mathcal{P} \rightarrow \mathcal{P}^{\prime}$ and a fibration $\mathcal{P}^{\prime} \rightarrow \mathcal{E}_{\mathcal{Q}}(M)$. Let $\mathcal{P}^{\prime \prime}$ be given by the pullback diagram

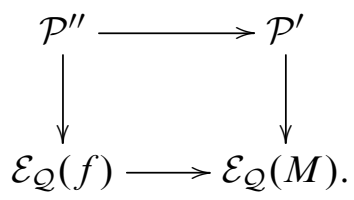

The upper map here is an equivalence because the lower one is. There are equivalences of pointed bimodule triples

$$
(\mathcal{P}, M, \mathcal{Q}) \rightarrow\left(\mathcal{P}^{\prime}, M, \mathcal{Q}\right) \leftarrow\left(\mathcal{P}^{\prime \prime}, M, \mathcal{Q}\right) \leftarrow\left(\mathcal{P}^{\prime \prime}, \mathcal{Q}, \mathcal{Q}\right),
$$

where the action of $\mathcal{P}^{\prime \prime}$ on $\mathcal{Q}$ arises from the operad map $\mathcal{P}^{\prime \prime} \rightarrow \mathcal{E}_{\mathcal{Q}}(f) \rightarrow \mathcal{Q}$. Since $E^{\mathrm{h}}\left(\mathcal{P}^{\prime \prime}, \mathcal{Q}, \mathcal{Q}\right)$ is distinguished by Proposition 6.3, Proposition 6.2 implies $E^{\mathrm{h}}(\mathcal{P}, M, \mathcal{Q})$ is also distinguished.

Proof of Theorem 1.9 We have to check Axioms I-VI from Section 3 in the case in which $\mathbf{C}$ is the category of graded spaces and $\diamond$ is the composition product. Theorem 3.11 then yields Theorem 1.9; the assumption that $\mathcal{O}_{1}$ is contractible guarantees that $\operatorname{Map}_{\mathbf{S}}\left(e_{\mathrm{O}}, \mathcal{O}\right) \sim \mathcal{O}_{1}$ is contractible.

Axiom I is Proposition 4.1; Axioms II and III are Propositions 5.1 and 6.1 respectively. Axiom IV(2) is Proposition 4.3 and Axiom V(2) follows as in Remark 3.7 from the fact that $e_{\circ}$ is cofibrant as a graded space. Axiom VI(1) is trivial, since $e_{\mathrm{O}}^{\mathrm{c}} \cong e_{\mathrm{O}}$. Finally, Axiom VI(2) is a consequence of the fact that if $\mathcal{P}$ is an operad, any cofibrant replacement $\mathcal{P}^{\mathrm{c}}$ for $\mathcal{P}$ as a $\mathcal{P}-\mathcal{P}$-bimodule is also cofibrant as a right $\mathcal{P}$-module by Lemma 6.4: given an operad map $\mathcal{P} \rightarrow \mathcal{Q}$, the functor $(-) \circ_{\mathcal{P}} \mathcal{Q}$ is left adjoint to the forgetful functor from right $\mathcal{Q}$-modules to right $\mathcal{P}$-modules; since the forgetful 
functor preserves equivalences and fibration, $(-) \circ_{\mathcal{P}} \mathcal{Q}$ preserves equivalences between cofibrant right $\mathcal{Q}$-modules.

\section{Classic monoids and the proof of Theorem 1.12}

A classic monoid is a monoid object in $(\mathbf{S p}, \times, *)$, ie, a simplicial semigroup with identity, or equivalently, an operad concentrated at level 1 . This last observation allows all of the results of the previous sections to be applied to classic monoids. With only notational changes, the same results (with the same proofs) hold for graded classic monoids, ie, monoid objects in $\left(\mathbf{S}, \odot, e_{\odot}\right)$.

The variant of Theorem 1.9 in which $\mathcal{A}$ is replaced by an arbitrary operad (the variant actually proved in Section 6) now translates to the following statement. Let $\mathbf{M}$ denotes the category of graded classic monoids.

7.1 Theorem Suppose that $\alpha: G \rightarrow H$ is a map of graded classic monoids, such that $H_{0} \sim *$. Then there is an equivalence

$$
\Omega \operatorname{Map}_{\mathbf{M}}^{\mathrm{h}}(G, H)_{\alpha} \sim \operatorname{Map}_{G-G}^{\mathrm{h}}(G, H),
$$

where on the right $H$ is treated as a $G-G$-bimodule via $\alpha$.

7.2 Remark The grading on these objects allows the assumption in 1.9 that the target operad is contractible at level 1 (which for ungraded classic monoids would translate into the unfortunate assumption that the target monoid is contractible) to be replaced by the assumption that the grade 0 constituent of the target graded monoid, in other words the constituent containing the identity element, is contractible.

A graded classic monoid is simply a left module over the associative operad $\mathcal{A}$, so specializing Theorem 7.1 to the case $G=\mathcal{A}$ gives the following result. For this statement, $A^{\prime}$ refers to the operad $\mathcal{A}$, treated as a left module over itself, ie, as a graded classic monoid.

7.3 Theorem Suppose that $\alpha: A^{\prime} \rightarrow X$ is a map of left $\mathcal{A}$-modules, and that $X_{0} \sim *$. Then there is an equivalence

$$
\Omega \operatorname{Map}_{\mathcal{A}}^{\mathrm{h}}\left(A^{\prime}, X\right)_{\alpha} \sim \operatorname{Map}_{A^{\prime}-A^{\prime}}^{\mathrm{h}}\left(A^{\prime}, X\right) .
$$

The mapping space on the right above is a derived mapping space of $\odot$-bimodules over the graded classic monoid $A^{\prime}$, where $X$ is treated as an $A^{\prime}-A^{\prime}$-bimodule via $\alpha$. Theorem 7.3 is remarkably similar to Theorem 1.12; the only difference being that, in 
Theorem 1.12, $A^{\prime}$ and $X$ have additional right $\mathcal{A}$-module structures, and the mapping spaces respect these additional structures. We will proceed to prove Theorem 1.12 by showing that introducing right $\mathcal{A}$-module structures does not materially change the arguments.

Some notation will be useful. As usual, $\mathbf{S}$ is the category of graded spaces, and for the purposes below $\mathbf{T}$ will denote the category of right $\mathcal{A}$-modules. We use $\mathbf{M}$ to denote the category of left $\mathcal{A}$-modules and $\mathbf{N}$ to denote the category of left $\mathcal{A}$-modules in $\mathbf{T}$ (ie, the category of $\mathcal{A}-\mathcal{A}$-bimodules). An object of $\mathbf{M}$ is a graded classic monoid, and an object of $\mathbf{N}$ is a graded classic monoid with a right $\mathcal{A}$-action compatible with the monoid multiplication.

If $G$ and $H$ belong to $\mathbf{N}$, we use the same letters to denote the underlying objects of $\mathbf{M}$. There are then categories $\mathbf{S}_{H}, \mathbf{T}_{H}, \mathbf{S}_{G, H}, \mathbf{T}_{G, H}$ of right $H$-modules or $G-H$-bimodules, as well as pointed variants $\mathbf{S}_{G, H}^{e}$ and $\mathbf{T}_{G, H}^{e}$. Keep in mind that these latter module structures refer to the graded cartesian product structure $\odot$, and that the basepoint for an object $X$ of $\mathbf{T}_{G, H}^{e}$, for instance, is a map $e_{\odot} \rightarrow X$, and so amounts to a point at level zero in the underlying graded space. Disregarding the right $\mathcal{A}$-module structure gives rise to forgetful functors: $\mathbf{T} \rightarrow \mathbf{S}, \mathbf{N} \rightarrow \mathbf{M}, \mathbf{T}_{H} \rightarrow \mathbf{S}_{H}$, $\mathbf{T}_{G, H} \rightarrow \mathbf{S}_{G, H}$, and $\mathbf{T}_{G, H}^{e} \rightarrow \mathbf{S}_{G, H}^{e}$.

7.4 Lemma All of the above forgetful functors are left adjoints, and so preserve colimits. All of the functors preserve (and reflect) equivalences, and preserve cofibrant objects.

Proof We will show that the functors preserve colimits, and the fact that they are left adjoints will be a consequence of the Adjoint Functor Theorem [16, V.6]. Let $U: \mathbf{A} \rightarrow \mathbf{B}$ be one of these forgetful functors. The functor $U$ preserves colimits if and only if it preserves coproducts and reflexive coequalizers; $U$ clearly preserves reflexive coequalizers, because as in Section 4.11 these can be computed in $\mathbf{S}$. Say that an object of $\mathbf{A}$ is free if it is in the image of the left adjoint $\Phi$ to the forgetful functor $F: \mathbf{A} \rightarrow \mathbf{S}$. Since every object $X$ of $\mathbf{A}$ is given a reflexive coequalizer $(\Phi F)^{2}(X) \Rightarrow \Phi F(X)$, in order to prove that $U$ preserves coproducts it is enough to show that $U$ preserves coproducts of free objects. But in each case this is clear by inspection, eg, for $U: \mathbf{T} \rightarrow \mathbf{S}$ and for free objects $X \circ \mathcal{A}$ and $Y \circ \mathcal{A}$ of $\mathbf{T}$,

$$
\begin{aligned}
U\left(X \circ \mathcal{A} \sqcup^{\mathbf{T}}(Y \circ \mathcal{A})\right) & \cong U\left(\left(X \sqcup^{\mathbf{S}} Y\right) \circ \mathcal{A}\right) \\
& \cong X \circ \mathcal{A} \sqcup^{\mathbf{S}} Y \circ \mathcal{A} .
\end{aligned}
$$

To put these isomorphisms in context, it is useful to observe that the functor $-\circ \mathcal{A}: \mathbf{S} \rightarrow \mathbf{S}$ preserves colimits, and that seen as a functor $\mathbf{S} \rightarrow \mathbf{S}_{\mathcal{A}}$, it creates colimits as well. 
It is clear that a map $f$ in $\mathbf{A}$ is an equivalence if and only if $U(f)$ is an equivalence. The statement about preservation of cofibrant objects follows from Remark 4.2 and an argument as above depending on the fact that $U$ preserves free objects.

Proof of Theorem 1.12 We have to check Axioms I-VI from Section 3 in the case in which $\mathbf{C}$ is the monoidal category $\left(\mathbf{T}, \odot, e_{\odot}\right)$; as above, we know that the axioms hold in $\left(\mathbf{S}, \odot, e_{\odot}\right)$. Theorem 3.11 will then yield Theorem 1.12; note that the assumption in Theorem 1.12 that $X_{0}$ is contractible guarantees that $\operatorname{Map}_{\mathbf{S}}\left(e_{\odot}, X\right)$ is contractible.

Axiom I follows as in 4.1 from [22, 7.1], since all of the categories involved are categories of algebras over $\mathbb{Z}^{+}$-sorted theories. The existence of the left adjoint required in Axiom III is standard. Suppose that $G$ and $H$ belong to $\mathbf{N}$; denote the coproduct in $\mathbf{N}$ of these objects by $G \sqcup H$. By Lemma 7.4, there is no harm in using the same notation for the coproduct of the images of $G$ and $H$ under the forgetful functor $\mathbf{N} \rightarrow \mathbf{M}$. There is a diagram of functors

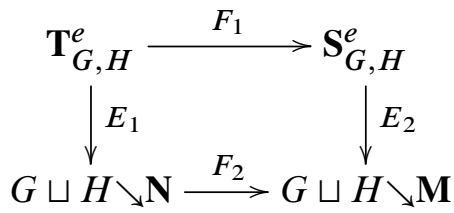

in which the horizontal arrows are forgetful functors and the vertical arrows are the appropriate left adjoints to forgetful functors. Adjointness gives a natural transformation $E_{2} F_{1} \rightarrow F_{2} E_{1}$. Since all of these functors commute with colimits by Lemma 7.4, to show that the diagram commutes up to natural isomorphism it is enough to show that the indicated natural transformation is an isomorphism when applied to a free object $G \odot\left(X_{+} \circ \mathcal{A}\right) \odot H$ of $\mathbf{T}_{G, H}^{e}$. (See the proof of Lemma 7.4; here $X \in \mathbf{S}$ and $X_{+}$is obtained by adding a disjoint basepoint at level 0 to $X$.) But the compositions $F_{2} E_{1}$ and $E_{2} F_{1}$ take this free object to $G \sqcup^{\mathbf{D}} H \sqcup^{\mathbf{D}}(\mathcal{A} \circ X \circ \mathcal{A})$, where $\mathbf{D}$ is respectively $\mathbf{N}$ or $\mathbf{M}$; by Lemma 7.4, the distinction between the two types of coproduct is irrelevant. The functor $F_{1}$ preserves equivalences distinguished objects, and cofibrant objects by Lemma 7.4, and so $E_{1}^{\mathrm{h}}$ preserves distinguished objects because $E_{2}^{\mathrm{h}}$ does. This verifies Axiom III.

The category $\mathbf{N}$ is left proper (and coproducts in $\mathbf{N}$ preserve equivalences) because of Lemma 7.4 and the fact that the corresponding statements hold in $\mathbf{M}$. This is Axiom IV(2). Axiom V(1) is immediate, because $e_{\odot}$ is cofibrant as a graded space. Axiom VI(1) is trivial, again because $e_{\odot}$ is cofibrant. Finally, Axiom VI(2) can be derived from combining the fact that the appropriate forgetful functor preserves coequalizers by Lemma 7.4 with the fact that the corresponding axiom holds in $\mathbf{M}$. 


\section{The main theorem}

Here we prove Theorem 1.1; we continue to use notation from Section 7 . The category $\mathbf{T}$ is the category of right $\mathcal{A}$ modules. The roman symbol $A$ denotes $\mathcal{A}$ in its role as a monoid in the monoidal category $\left(\mathbf{T}, \odot, e_{\odot}\right)$, and $\mathbf{T}_{A, A}$ is the category of $A-A$ bimodules in $\mathbf{T}$.

Suppose that $\mathcal{O}$ is a reduced operad and that $\omega: \mathcal{A} \rightarrow \mathcal{O}$ is an operad map. Combining Theorem 1.9 with Theorem 1.12 gives an equivalence

$$
\Omega^{2} \operatorname{Map}_{\mathbf{O}}^{\mathrm{h}}(\mathcal{A}, \mathcal{O})_{\omega} \sim \operatorname{Map}_{\mathbf{T}_{A, A}}^{\mathrm{h}}(A, \mathcal{O}) .
$$

We will calculate the mapping space on the right by using the following observation.

8.2 Proposition The category $\mathbf{T}_{A, A}$ is equivalent to the category of cosimplicial spaces.

More explicitly, to give a graded space $X=\left\{X_{n}\right\}$ the structure of an $A-A$ bimodule in the category of right $\mathcal{A}$ modules is equivalent to providing a collection of cosimplicial operators

$$
\begin{array}{cll}
d^{i}: X_{n} \rightarrow X_{n+1}, & n \geq 0, & 0 \leq i \leq n+1, \\
s^{i}: X_{n} \rightarrow X_{n-1}, & n \geq 1, & 0 \leq i \leq n-1
\end{array}
$$

satisfying the usual cosimplicial identities. Verifying this is a routine exercise: given the indicated structure on $X$, the cosimplicial operators are extracted as follows.

$$
\begin{array}{cr}
d^{0}: A_{1} \times X_{n} \rightarrow X_{n+1} & \text { left } A \text { action, } \\
d^{1 \leq i \leq n}: X_{n} \times \mathcal{A}_{1}^{i-1} \times \mathcal{A}_{2} \times \mathcal{A}_{1}^{n-i} \rightarrow X_{n+1} & \text { right } \mathcal{A} \text { action, } \\
d^{n+1}: X_{n} \times A_{1} \rightarrow X_{n+1} & \text { right } A \text { action, } \\
s^{i}: X_{n} \times \mathcal{A}_{1}^{i} \times \mathcal{A}_{0} \times \mathcal{A}_{1}^{n-i-1} \rightarrow X_{n-1} & \text { right } \mathcal{A} \text { action. }
\end{array}
$$

If $X$ is an operad $\mathcal{O}$ with a map $\mathcal{A} \rightarrow \mathcal{O}$, treated as an object of $\mathbf{T}_{A, A}$ along the lines of Theorems 1.9 and 1.12, these operators provide the cosimplicial object $\mathcal{O}^{\bullet}$ constructed in [19, Section 3] by McClure and Smith (cf Section 1.2).

The proof of Theorem 1.1 is immediate. Let CS be the category of cosimplicial spaces. According to the above remarks, the right hand side of (8.1) can be rewritten as

$$
\operatorname{Map}_{\mathbf{T}_{A, A}}^{\mathrm{h}}(A, \mathcal{O})=\operatorname{Map}_{\mathbf{C S}}^{\mathrm{h}}\left(*, \mathcal{O}^{\bullet}\right),
$$

where $*$ denotes the constant cosimplicial space with a point in each cosimplicial degree. The model category structure on $\mathbf{T}_{A, A}$ with respect to which $\operatorname{Map}{ }^{\mathrm{h}}(-,-)$ is 
formed represents a simplicial model structure on CS in which the equivalences are determined levelwise; according to [6], this choice of equivalences is the only aspect of the model category structure which affects the weak homotopy type of $\operatorname{Map}^{\mathrm{h}}(-,-)$. But Bousfield and Kan define the homotopy limit of a diagram $Y$ of spaces, in terms of such an object $\operatorname{Map}^{\mathrm{h}}(*, Y)$; more specifically, they define holim $Y$ as $\operatorname{Map}\left(*^{\mathrm{c}}, Y^{\mathrm{f}}\right)$, where $*^{\mathrm{c}}$ is a cofibrant replacement for $*, Y^{\mathrm{f}}$ is a fibrant replacement for $Y$, and the work is done within a simplicial model structure on the diagram category in which equivalences are determined objectwise [2, XI.8].

\section{References}

[1] J Adámek, J Rosický, E M Vitale, Algebraic theories, preprint (2008) Available at http://www.iti.cs.tu-bs.de/ adamek/algebraic.theories.pdf

[2] A K Bousfield, D M Kan, Homotopy limits, completions and localizations, Lecture Notes in Math. 304, Springer, Berlin (1972) MR0365573

[3] D Dugger, Classification spaces of maps in model categories arXiv: math.AT/0604537

[4] W G Dwyer, PS Hirschhorn, D M Kan, J H Smith, Homotopy limit functors on model categories and homotopical categories, Math. Surveys and Monogr. 113, Amer. Math. Soc. (2004) MR2102294

[5] W G Dwyer, D M Kan, Calculating simplicial localizations, J. Pure Appl. Algebra 18 (1980) 17-35 MR578563

[6] W G Dwyer, D M Kan, Function complexes in homotopical algebra, Topology 19 (1980) 427-440 MR584566

[7] W G Dwyer, D M Kan, Simplicial localizations of categories, J. Pure Appl. Algebra 17 (1980) 267-284 MR579087

[8] PG Goerss, J F Jardine, Simplicial homotopy theory, Progress in Math. 174, Birkhäuser, Basel (1999) MR1711612

[9] TG Goodwillie, Excision estimates for spaces of homotopy equivalences, preprint (1995) Available at http://www.math.brown.edu/ tomg/ excisionhtpytex.pdf

[10] T G Goodwillie, J R Klein, Multiple disjunction for spaces of Poincaré embeddings, J. Topol. 1 (2008) 761-803 MR2461855

[11] T G Goodwillie, M Weiss, Embeddings from the point of view of immersion theory: Part II, Geom. Topol. 3 (1999) 103-118 MR1694808

[12] PS Hirschhorn, Model categories and their localizations, Math. Surveys and Monogr. 99, Amer. Math. Soc. (2003) MR1944041 
[13] M Hovey, Model categories, Math. Surveys and Monogr. 63, Amer. Math. Soc. (1999) MR1650134

[14] M Kontsevich, Operads and motives in deformation quantization, Lett. Math. Phys. 48 (1999) 35-72 MR1718044 Moshé Flato (1937-1998)

[15] A Lazarev, Spaces of multiplicative maps between highly structured ring spectra, from: "Categorical decomposition techniques in algebraic topology (Isle of Skye, 2001)", (G Arone, J Hubbuck, R Levi, M Weiss, editors), Progr. Math. 215, Birkhäuser, Basel (2004) 237-259 MR2039769

[16] S Mac Lane, Categories for the working mathematician, second edition, Graduate Texts in Math. 5, Springer, New York (1998) MR1712872

[17] J P May, The geometry of iterated loop spaces, Lectures Notes in Math. 271, Springer, Berlin (1972) MR0420610

[18] J P May, Simplicial objects in algebraic topology, Chicago Lectures in Math., Univ. of Chicago Press (1992) MR1206474

[19] J E McClure, J H Smith, A solution of Deligne's Hochschild cohomology conjecture, from: "Recent progress in homotopy theory (Baltimore, MD, 2000)", (D M Davis, J Morava, G Nishida, W S Wilson, N Yagita, editors), Contemp. Math. 293, Amer. Math. Soc. (2002) 153-193 MR1890736

[20] D G Quillen, Homotopical algebra, Lecture Notes in Math. 43, Springer, Berlin (1967) MR0223432

[21] C Rezk, Spaces of algebra structures and cohomology of operads, PhD thesis, Massachusetts Institute of Technology (1996) Available at http://www.math.uiuc.edu/ rezk/rezk-thesis.dvi

[22] C Rezk, Every homotopy theory of simplicial algebras admits a proper model, Topology Appl. 119 (2002) 65-94 MR1881711

[23] D P Sinha, Operads and knot spaces, J. Amer. Math. Soc. 19 (2006) 461-486 MR2188133

[24] M Weiss, Embeddings from the point of view of immersion theory: Part I, Geom. Topol. 3 (1999) 67-101 MR1694812

Department of Mathematics, University of Notre Dame

Notre Dame IN 46556, USA

Institut de Géométrie, algèbre et topologie (IGAT), École Polytechnique Fédérale de Lausanne CH-1015 Lausanne, Switzerland

dwyer.1@nd.edu, kathryn.hess@epfl.ch

http://www.nd.edu/ wgd, http://sma.epfl.ch/ hessbell/

Proposed: Paul Goerss

Seconded: Haynes Miller, Ralph Cohen
Received: 7 December 2010

Revised: 6 February 2012 